\title{
Fokker-Planck simulations of fast flows of melts and concentrated polymer solutions in complex geometries
}

\author{
Alexei Lozinski \\ LMF-ISE-FSTI, Ecole Polytechnique Fédérale de Lausanne, CH 1015 \\ Lausanne, Switzerland \\ Cédric Chauvière \\ Division of Applied Mathematics, Brown University, Box F, Providence, \\ Rhode Island 02912 \\ Jiannong Fang
}

GEOLEP-ICARE-FENAC, Ecole Polytechnique Fédérale de Lausanne, CH 1015

Lausanne, Switzerland

Robert G. Owens ${ }^{\text {a) }}$

LMF-ISE-FSTI, Ecole Polytechnique Fédérale de Lausanne, CH 1015

Lausanne, Switzerland

(Received 19 September 2002; final revision received 18 December 2002)

\section{Synopsis}

In 1999, Öttinger introduced a thermodynamically admissible reptation model incorporating chain stretching, anisotropic tube cross sections, double reptation, and the convective constraint release mechanism. In this paper, we describe and use a new high-order Fokker-Planck-based numerical method for the simulation of the Öttinger model in complex geometries. Evidence, in the case of startup homogeneous flows, of the significant CPU time advantage (for comparable levels of accuracy) of our method over a stochastic simulation [Fang et al. (2000)], is presented. For the confined cylinder benchmark problem, differences in the drag behavior observed between the Öttinger model and those of Doi and Edwards (1978a, 1978b, 1978c) and Mead et al. (1998) are explained in terms of double reptation and the differing relaxation spectra. (C) 2003 The Society of Rheology. [DOI: 10.1122/1.1545440]

\section{INTRODUCTION}

Our concern in this paper is the accurate, yet cost-effective, simulation of "fast" flows of melts and concentrated polymer solutions in complex geometries. By concentrated polymer solutions we are referring to those solutions of polymers where faithful constitutive modeling requires that interactions between polymers in their solution be taken into account. In this context the best known coarse-grained description of the interaction of a polymer molecule with the surrounding matrix is provided by so-called reptation models,

"Author to whom all correspondence should be addressed; electronic mail: Robert.Owens@epfl.ch 
originally introduced by de Gennes (1971) and extended by Doi and Edwards (1978a, $1978 \mathrm{~b}, 1978 \mathrm{c}$ ) in three landmark papers of the late 1970s. In these models a polymer molecule moves through a tube whose surface is formed by the surrounding polymers. In the interests of tractability Doi and Edwards ignored the connectivity of the reptating chain and thereby reduced the model to a single segment description. Furthermore, they introduced the independent alignment assumption (IAA). Since after deformation the polymer molecules in the Doi-Edwards (DE) model retract instantaneously back to their equilibrium length, stretching of polymer chains (or of their surrounding tubes) was excluded. The prediction of excessive shear thinning in fast steady shear flows is one of the unrealistic features of the DE theory. As explained in numerous papers [see, for example those by Mead et al. (1998), Fang et al. (2000), and Peters et al. (2000b)], this particular weakness is not overcome by building in a nonzero retraction time, and thus allowing tube stretch: the polymers simply end up by orientating themselves along the flow direction and the drag on them is reduced as a consequence. The crucial difference between modern reptation theory [e.g., Marrucci (1996); Ianniruberto and Marrucci (1996); Mead et al. (1998)] and that of Doi and Edwards lies elsewhere: for sufficiently fast flows proper account is taken in these models of a release of constraints by motion of the members of the matrix that forms the tube around a given polymer chain. Thus, the polymer is far freer to relax than would be the case by reptation alone. This convective constraint release (CCR) mechanism suppresses the tendency of polymer chains to align with a shear flow and occurs when polymers of the surrounding matrix move faster than the polymer chain within is able to relax, i.e., for shear rates $\dot{\gamma}>\tau_{d}^{-1}$, where $\tau_{d}$ denotes the reptation time.

In 1999 Öttinger introduced a thermodynamically admissible reptation model incorporating chain stretching, anisotropic tube cross sections, double reptation [Tsenoglu (1987); des Cloizeaux (1988)], and the CCR mechanism. In addition, as with other modern reptation models Öttinger avoided the IAA. The constraint release mechanisms associated with double reptation and CCR were incorporated in a natural way into the dissipative part of the new model. Two versions of the model were proposed: a "uniform" model where the chain contour label $s$ was uninfluenced by the flow field so that only uniform stretching of the chain could occur, and a "tuned" model where $s$ was rescaled by the total tube stretching rate. A year later Fang et al. (2000) used a stochastic method to evaluate the uniform model in various transient and steady shear and extensional flows by comparing the predictions of this model with experimental results [for a solution of polystyrene in tricresyl phosphate, Kahvand (1995)] and with the predictions of two other reptation models [Hua and Schieber (1998); Hua et al. (1999); Mead et al. (1998)]. Anisotropic tube cross sections were not considered. In the shear flows the three reptation models were seen to manifest similar behavior in many cases and the Öttinger model was able to capture, at least qualitatively, the real polymer behavior. In a steadystate uniaxial extensional flow Fang et al. observed the appearance of a low extensional rate region $0.1 \tau_{d}^{-1} \leqslant \dot{\epsilon} \leqslant \tau_{d}^{-1}$ where the viscosity manifested extensional hardening. It was thought at the time that this represented a difference in the extensional behavior with that of the DE and Mead-Larson-Doi (MLD) models. This is now known not to be the case [Hassager (2002)].

The purpose of this paper is twofold: the evaluation, for the first time, of the uniform Öttinger (UÖ) model in a complex geometry and the showcasing of Fokker-Planck (FP)based numerical methods as a competitive alternative to stochastic methods for the simulation of fast flows of melts and concentrated polymer solutions.

The basic idea of the micro-macro approach is to combine the usual numerical solution to the (macroscopic) conservation equations with a kinetic theory model for the 
polymeric contribution to the extra-stress tensor. Thus, closed-form differential or integral constitutive equations may be circumvented and Itô-type stochastic differential equations solved instead for the conformations of the polymer molecules describing the coarse-grained (mesoscopic-level) microstructure. Macroscopic quantities, for example the elastic stress, may then be computed by averaging large ensembles of realizations of the conformations [see the book of Öttinger (1996) for details]. Over the decade or so that has elapsed since the pioneering work of Laso and Öttinger (1993) with the CONNFFESSIT method, micro-macro simulations have become increasingly popular, primarily because of the exciting possibilities that have opened up for incorporating more of the essential physics of polymeric fluids into the kinetic theory models used in such simulations. The Brownian configuration field method [Hulsen et al. (1997); Öttinger et al. (1997)] and the Lagrangian particle method [Halin et al. (1998); Wapperom et al. (2000)] define, in our opinion, the current state of the art.

The starting point of FP-based methods is the equivalence that exists between a stochastic differential equation and the FP equation that describes the distribution of the stochastic process. Since the aim in view in the "micro" part of micro-macro methods is usually to compute a macroscopic elastic stress, clearly one does not need to calculate the trajectories of individual conformations, as is done in a CONNFFESSIT-type method, for example. The determination of a configuration probability density function (pdf) is quite sufficient for the computation of ensemble averages in configurational space of functions of the polymer conformation, and thus for finding the macroscopic quantities of interest. However, coolness to the idea of solving an equivalent FP equation for the polymeric configuration pdf, at least in the case of a high-dimensional configurational space, has been expressed in the literature [Öttinger (1996), Owens and Phillips (2002)], on the grounds of computational cost.

Indeed, very little has been done to advocate FP-based methods as a convenient way of computing a macroscopic elastic stress. Exceptions are the papers of Warner (1972) and Fan $(1985 \mathrm{a}, 1985 \mathrm{~b}, 1985 \mathrm{c})$ on steady-state shearing flows and small amplitude oscillatory shear flows of dumbbell models. Fan (1989a) also computed the material functions for steady-state shear flow and uniaxial extensional flow of rigid rod-like molecular suspensions. In part II of the paper, Fan (1989b) performed what, to our knowledge at least, were the first computations in the published literature of complex flows using the FP equation. In recent work by the MIT group [Armstrong et al. (1996); Nayak (1998); Suen et al. (2002)] the discontinuous Galerkin method has been used for spatially discretizing the FP equation for dumbbell and DE models and a Daubechies wavelet basis employed for representations in configurational space.

In Sec. II we give a mathematical description of the three reptation models that will be compared in low Deborah number flow past a confined cylinder in Sec. IV B 1. Section III is devoted to an exposition of our spectral/spectral element method for the discretization of the conservation equations for mass and linear momentum, the evolution equation for the tube stretch parameter $\lambda$ and the FP equation for the configuration pdf $\psi$. The crucial element in the numerical algorithm is a time-splitting method for the equations for $\lambda$ and $\psi$, permitting resolution first in configurational space and then in physical space. In Secs. IV A 1 and IV A 2 we compare the results obtained using our FP-based method for the UÖ model in startup homogeneous flows with those computed using the stochastic method of Fang et al. (2000). A clear computational advantage in favor of the FP-based approach is evidenced. In the last subsection the UÖ model is evaluated in a complex flow for the first time. We consider the behavior of this model compared with that of the single-mode DE model and Mead-Larson-Doi [Mead et al. (1998)] model in flow past a 
cylinder at low Deborah numbers, the data for the DE and MLD models being taken from the paper of Peters et al. (2000b). Some conclusions follow.

\section{DESCRIPTION OF THE MODELS}

The equations of continuity and of linear momentum for an incompressible polymer solution in inertialess and isothermal flow are

$$
\boldsymbol{\nabla} \cdot \mathbf{v}=0
$$

and

$$
\mathbf{0}=-\nabla p+\eta_{s} \nabla^{2} \mathbf{v}+\nabla \cdot \boldsymbol{\tau}+\mathbf{f}
$$

where $\mathbf{v}, p$, and $\boldsymbol{\tau}$ denote, respectively, the velocity, pressure, and elastic stress fields. $\eta_{s}$ in Eq. (2) is the viscosity of the Newtonian solvent and $\mathbf{f}$ is a body force (e.g., gravity).

Our interest in this paper is the numerical simulation of melts and concentrated polymer solutions in which the elastic contribution to the Cauchy stress is derivable from a reptation model. Let us denote by $\mathbf{u}$ the unit orientation vector of a tube segment and by $s \in[0,1]$ the normalized contour label of a polymer chain, such that $s=0$ and $s=1$ correspond to the head and tail, respectively, of the polymer chain. Then, a configuration probability density function $\psi(\mathbf{u}, s, \mathbf{x}, t)$ may be introduced, so that $\psi(\mathbf{u}, s, \mathbf{x}, t) d \mathbf{u} d s$ is the joint probability that at time $t$ a tube segment with position vector $\mathbf{x}$ has associated orientation vector in the interval $[\mathbf{u}, \mathbf{u}+d \mathbf{u}]$ and contains the part of the polymer chain labeled in the interval $[s, s+d s]$. In this paper we describe three reptation models: the DE model [Doi and Edwards (1978a, 1978b, 1978c)], the MLD model [Mead et al. (1998)], and the UÖ model [Öttinger (1999)]. Unlike the DE model, the reptation models of Mead et al. and Öttinger allow for tube stretching and both incorporate CCR. Additionally, the UÖ model has two features not present in the MLD model: double reptation [see Eq. (18) and the succeeding paragraph] and the possibility of incorporating anisotropy in the tube cross sections. As with Fang et al. (2000), however, we make no use in this paper of this last feature of Öttinger's model.

\section{A. Doi-Edwards model}

One of the most basic reptation models and the spawning mother of all the rest is the original DE model [Doi and Edwards (1978a, 1978b, 1978c)] with the IAA. In this form the configuration pdf is the solution to the FP equation

$$
\frac{D \psi}{D t}=-\frac{\partial}{\partial \mathbf{u}} \cdot[(\mathbf{I}-\mathbf{u u}) \cdot \boldsymbol{\kappa} \cdot \mathbf{u} \psi]+\frac{1}{\pi^{2} \tau_{d}} \frac{\partial^{2} \psi}{\partial s^{2}},
$$

where $\boldsymbol{\kappa}$ denotes the transposed velocity gradient tensor $\left(\kappa_{i j}=\partial v_{i} / \partial x_{j}\right), D / D t$ is the material derivative, and $\tau_{d}$ is the reptation time, defined as the characteristic time for a polymer chain to escape from its original tube. The differential operator $\partial / \partial \mathbf{u}$ in $\mathrm{Eq}$. (3) includes only the derivatives tangent to the unit sphere. The boundary conditions for $s=0$ and $s=1$ supplementing the FP equation (3) are

$$
\psi(\mathbf{u}, s, \mathbf{x}, t)=\frac{1}{4 \pi} \delta(|\mathbf{u}|-1), \quad s=0,1,
$$

where $\delta$ denotes the delta function. The elastic contribution $\tau$ to the Cauchy stress is then determined in the Doi-Edwards theory by

$$
\boldsymbol{\tau}=5 G_{N}^{0} \mathbf{S}
$$


where $G_{N}^{0}$ is an elastic modulus, $\mathbf{S}=\langle\mathbf{u u}\rangle$ is the orientation tensor and $\langle\cdot\rangle$ denotes an ensemble average, defined for a function $f=f(\mathbf{u}, s)$ by

$$
\langle f\rangle=\int_{s=0}^{1} \int_{B(0,1)} f(\mathbf{u}, s) \psi(\mathbf{u}, s, \mathbf{x}, t) d \mathbf{u} d s
$$

$B(0,1)$ being the surface of the unit sphere centered at the origin.

We note that the DE model can be written in equivalent form as an integral equation for the orientation tensor $\mathbf{S}(t)$ :

$$
\mathbf{S}(t)=\int_{t^{\prime}=-\infty}^{t} m\left(t, t^{\prime}\right) \mathbf{Q}\left[\mathbf{F}\left(t, t^{\prime}\right)\right] d t^{\prime},
$$

where $m$ is the memory function, given for the DE model by

$$
m\left(t, t^{\prime}\right)=m^{\mathrm{DE}}\left(t, t^{\prime}\right)=\frac{8}{\pi^{2} \tau_{d}} \sum_{k}^{\infty} \exp \left(-\frac{(2 k+1)^{2}\left(t-t^{\prime}\right)}{\tau_{d}}\right),
$$

and $\mathbf{Q}$ is the deformation-dependent tensor defined in terms of the deformation gradient tensor $\mathbf{F}\left(t, t^{\prime}\right)$ as follows:

$$
\mathbf{Q}\left(t, t^{\prime}\right)=\langle\mathbf{u u}\rangle_{t^{\prime}}=\left\langle\frac{\mathbf{F}\left(t, t^{\prime}\right) \cdot \mathbf{u}\left(t^{\prime}\right) \mathbf{F}\left(t, t^{\prime}\right) \cdot \mathbf{u}\left(t^{\prime}\right)}{\left|\mathbf{F}\left(t, t^{\prime}\right) \cdot \mathbf{u}\left(t^{\prime}\right)\right|^{2}}\right\rangle_{t^{\prime}}
$$

The subscript $t^{\prime}$ in Eq. (9) indicates that the orientation vector $\mathbf{u}$ in the ensemble average was created at time $t^{\prime}$ according to the uniform distribution as in the boundary conditions (4). The integration in Eq. (7) is performed along the particle paths. The DE simulations of Peters et al. (2000) using a deformation field method, the results of which are discussed in Sec. IV B 1, were for a single-mode $(\mathrm{sm})$ version of this model. In this case the memory function is given by

$$
m^{\mathrm{DEsm}}\left(t, t^{\prime}\right)=\frac{1}{\tau_{d}} \exp \left(-\frac{\left(t-t^{\prime}\right)}{\tau_{d}}\right)
$$

and the corresponding FP equation by

$$
\frac{D \psi}{D t}=-\frac{\partial}{\partial \mathbf{u}} \cdot[(\mathbf{I}-\mathbf{u u}) \cdot \boldsymbol{\kappa} \cdot \mathbf{u} \psi]-\frac{1}{\tau_{d}}\left(\psi-\frac{1}{4 \pi}\right) .
$$

As mentioned already in the Introduction, although the predictions of the DE model are in excellent agreement with experimental step-shear strain data, the model is severely shear thinning in steady shear flow. Some more recent reptation models incorporate two important improvements to the original DE model: tube stretching for flows faster than the inverse of the Rouse time $\tau_{s}$ for the relaxation of the polymer chain, and the CCR mechanism for flows faster than the inverse of the reptation time $\tau_{d}$. Among such models we cite ones by Marrucci (1996), Ianniruberto and Marrucci (1996), Mead et al. (1998), and the UÖ model [Öttinger (1999), Fang et al. (2000)]. In the following subsections we recapitulate the essential mathematics of the latter two models.

\section{B. Mead-Larson-Doi model}

The MLD model is based on the model by Pearson et al. (1989), which is a simplified form of the Marrucci-Grizzuti extension of the DE model [Marrucci and Grizzuti 
(1988)]. The MLD model incorporates the effects of tube stretching and constraint release. Specifically, account is taken of tube stretching via a function $\lambda=\lambda(\mathbf{x}, t)$ defined as

$$
\lambda=\frac{L}{L_{0}}
$$

where $L$ is the contour length of a polymer chain and $L_{0}$ its equilibrium contour length. $\lambda$ is incorporated into the expression for $\tau$ as

$$
\tau=5 G_{N}^{0} \lambda^{2} S
$$

where $\mathbf{S}=\langle\mathbf{u u}\rangle$ is the orientation tensor, which is calculated through the integral equation (7) with the memory function specified below. The time rate of change of $\lambda$ is governed by the balance between stretch due to the flow field and relaxation of the chain within the tube. Constraint release effects are also taken into account, which gives the equation

$$
\dot{\lambda}=\frac{D \lambda}{D t}=\lambda \boldsymbol{\kappa}: \mathbf{S}-\frac{1}{\tau_{s}}(\lambda-1)-\frac{1}{2}\left(\boldsymbol{\kappa}: \mathbf{S}-\frac{\dot{\lambda}}{\lambda}\right)(\lambda-1),
$$

where $\tau_{s}$ is the longest Rouse time of the polymer chain.

In deriving the expression for the memory function, Mead et al. started from the memory function of the single-mode DE model (10). The latter may be seen to satisfy

$$
\frac{D m^{\mathrm{DEsm}}}{D t}=-\frac{1}{\tau_{d}} m^{\mathrm{DEsm}}
$$

with an effective relaxation time $\tau=\tau_{d}$. By modifying $\tau$ to take account of an extra relaxation of orientation due to CCR, Mead et al. proposed a memory function that satisfies

$$
\frac{D m^{\mathrm{MLD}}}{D t}=-\left[\frac{1}{\lambda^{2} \tau_{d}}+\frac{1}{\lambda}\left(\boldsymbol{\kappa}: \mathbf{S}-\frac{\dot{\lambda}}{\lambda}\right)\right] m^{\mathrm{MLD}} .
$$

Here, the reptation time $\tau_{d}$ is multiplied by $\lambda^{2}$ to take account of the increase in the reptation time produced as a result of the lengthening of the chain's primitive path by the flow.

\section{Uniform Öttinger model}

The reptation models described by Öttinger (1999) feature anisotropic tube cross sections, chain stretching, double reptation, and CCR, while avoiding the IAA. In his paper Öttinger discussed two versions of the model: a "uniform" model where the contour label $s$ is uninfluenced by the flow (and thus only a uniform stretching of the chain can occur), and a "tuned" model where $s$ is rescaled by the total chain stretching rate. In Part II of the paper, Fang et al. (2000) performed stochastic simulations of the uniform model for various shear and extensional flows, but made no use of anisotropy in the tube cross sections. With an eye to the numerical results in Sec. IV of the present paper we now briefly discuss the equations characterizing the UÖ model, as implemented by Fang et al.

The model features a maximum allowable extension ratio $\lambda_{\max }$ and the evolution equation for the chain stretching ratio $\lambda$ is

$$
\dot{\lambda}=\frac{D \lambda}{D t}=\lambda \kappa: S-\frac{1}{\tau_{s}} \frac{\left(\lambda^{2}-1\right) \lambda_{\max }^{2}}{\lambda\left(\lambda_{\max }^{2}-\lambda^{2}\right)} .
$$


The first term on the right-hand side of Eq. (16) corresponds to affine deformation and will be denoted in Sec. III by $\dot{\lambda}_{\text {con }}$. The second term is a dissipative contribution, denoted hereafter by $\dot{\lambda}_{\text {dissip }}$.

The FP equation for the configuration pdf assumes the form

$$
\frac{D \psi}{D t}=-\frac{\partial}{\partial \mathbf{u}} \cdot[(\mathbf{I}-\mathbf{u u}) \cdot \boldsymbol{\kappa} \cdot \mathbf{u} \psi]-\frac{\partial}{\partial s}\left(\dot{s}_{\text {tot }} \psi\right)-\frac{\dot{\lambda}_{\text {dissip }}}{\lambda} \psi+\frac{1}{\pi^{2} \tau_{d}} \frac{\partial^{2} \psi}{\partial s^{2}}+D \frac{\partial}{\partial \mathbf{u}} \cdot \frac{\partial \psi}{\partial \mathbf{u}},
$$

where the drift velocity $\dot{s}_{\text {tot }}=-1 / \lambda[s-(1 / 2)] \dot{\lambda}_{\text {dissip }}$ and $D$ is an orientational diffusion coefficient. The second term on the right-hand side of Eq. (17) is a creation/destruction term accounting for nonzero drift of configurations through the boundaries $s=0$ and $s=1$. The boundary conditions for the FP equation (17) are the same as those in Eq. (4). The physical significance of the terms in Eq. (17) is discussed by Öttinger (1999) and by Fang et al. (2000). The orientational diffusion coefficient $D$ in Eq. (17) may be defined as

$$
D=\frac{1}{6}\left[\delta_{1} \frac{1}{\tau_{d}}-\delta_{2} \frac{\dot{\lambda}_{\text {dissip }}}{\lambda} H\left(-\frac{\dot{\lambda}_{\text {dissip }}}{\lambda}\right)\right]
$$

where $H$ is a Heaviside function. $\delta_{1}$ and $\delta_{2}$ are positive $\lambda$-dependent parameters representing double reptation and the CCR mechanism, respectively. Double reptation is so called because with $\delta_{1}=1$ there is an additional relaxation mechanism with the same reptation time as in the original DE model. Following Fang et al. (2000) we have chosen $\delta_{1}=\delta_{2}=1 / \lambda$.

Finally, the elastic stress $\boldsymbol{\tau}$ is related to the orientation tensor $\mathbf{S}=\langle\mathbf{u u}\rangle$ by

$$
\boldsymbol{\tau}=5 G_{N}^{0}\left[1+\frac{\lambda^{2}-1}{1-\left(\lambda / \lambda_{\max }\right)^{2}}\right] \mathbf{S}
$$

and is the sum of a DE contribution [first term in the square parentheses of Eq. (19)] and a contribution associated with chain stretching. We note in passing that as $\lambda_{\max } \rightarrow \infty$ $\tau \rightarrow 5 G_{N}^{0} \lambda^{2} S$, which is the same as Eq. (12).

In the presentation of the results in Sec. IV we work with dimensionless variables, here denoted with an asterisk, and defined by

$$
\mathbf{x}^{*}=\mathbf{x} / L, t^{*}=t / \tau_{d}, \quad \mathbf{v}^{*}=\mathbf{v} \tau_{d} / L, \quad p^{*}=p / G_{N}^{0}, \quad \text { and } \tau^{*}=\tau / G_{N}^{0},
$$

where $L$ represents a suitably chosen length scale.

In rewriting Eqs. (1) and (2), (16) and (17), and (19) in terms of these dimensionless variables, it may be shown easily that the only parameters that require prescription are the maximum extension ratio $\lambda_{\max }$, the ratio of the reptation time to the Rouse time $\tau_{d} / \tau_{s}$, and the dimensionless solvent viscosity $\eta_{s}^{*}=\eta_{s} / G_{N}^{0} \tau_{d}$.

\section{NUMERICAL METHOD}

One of the most efficient techniques that has been used up to now for numerical simulation of reptation models in complex flows is the deformation field method [Peters et al. (2000a); Hulsen et al. (2001)]. It is applied by van Heel et al. (1999) to the DE model and by Peters et al. (2000b) to the MLD model. This method is based on the integral representations (7) and (9) of the orientation tensor $\mathbf{S}(t)$. However, such a simple representation does not exist for the UÖ model due to the presence of the second and 
third terms on the right-hand side of its FP equation (17). This makes impossible the application of the deformation field method to the UÖ model.

An alternative is to use stochastic numerical methods, [see, for example, the book of öttinger (1996)]. This approach is based on the equivalence of FP equations and stochastic differential equations and was applied to the UÖ model by Fang et al. (2000). However, only homogeneous flows were considered by these authors.

Very recently, Gigras and Khomami (2002) have combined the Brownian configuration field method and the deformation field method to allow the simulation of advanced reptation models such as the UÖ model. Their so-called adaptive configuration field method was tested against the results of the Brownian dynamics simulations of Fang et al. (2000) for several homogeneous flows and the results were found to be in excellent agreement.

In the present paper we explore an alternative approach to those cited above: solving directly the FP equation (17) using high-order methods. We shall show that this can be much more efficient that stochastic methods in the case of homogeneous flows, and moreover complex flows can also be investigated. The equations to solve in the UÖ model are Eqs. (1), (2), (16), and (17). This necessitates discretization in time and in both configurational and physical space. A Galerkin method is used in configurational space and a streamline-upwinded Petrov/Galerkin (SUPG) spectral element method in physical space, as will be elaborated on in the subsections to follow.

\section{A. Time-splitting scheme}

We denote the evaluation of a field variable $(\mathbf{v}, p, \tau, \lambda, \psi)$ at time $t=j \Delta t$ with a superscript " $j . "$

The initial conditions $(j=0)$ are chosen as $\tau^{0}=\mathbf{0}, \lambda^{0}=1$, and $\psi^{0}=1 / 4 \pi$. For simplicity, we decouple the solution of the Stokes system (1) and (2) from the polymeric stress calculations (16), (17), and (19). Thus, $\boldsymbol{\tau}^{j}$ appears as a source term evaluated at time $j \Delta t$ in Eq. (2) and we solve the continuity-momentum pair for velocity $\mathbf{v}^{j}$ and pressure $p^{j}$ :

$$
\begin{aligned}
& \boldsymbol{\nabla} \cdot \mathbf{v}^{j}=0, \\
& \boldsymbol{\nabla} p^{j}-\eta_{s} \boldsymbol{\nabla}^{2} \mathbf{v}^{j}=\boldsymbol{\nabla} \cdot \boldsymbol{\tau}^{j} .
\end{aligned}
$$

Since the FP equation (17) contains derivatives in both configurational and physical spaces, solving it in a completely implicit way would be prohibitively expensive. On the other hand, it is better to treat the convective terms in physical space implicitly for stability reasons. As a compromise, we propose splitting every time step into two halftime steps, the first one (explicit) accounting for configurational space and the second one (implicit) for physical space. Moreover, the first half-time step can be further split in $N_{\alpha}$ smaller time steps to meet possible restrictions of Courant-Friedrichs-Lewy type. $N_{\alpha}$ was set equal to 10 for all the results presented in Sec. IV. All this leads to the following time marching scheme:

- First half-time step: mulas

Set $\lambda^{j(0)}=\lambda^{j}$ and $\psi^{j(0)}=\psi^{j}$ and calculate $\psi^{j(\alpha)}$ for $\alpha=0, \ldots, N_{\alpha}-1$ via the for-

$$
\frac{\lambda^{j(\alpha+1)}-\lambda^{j(\alpha)}}{\Delta t / N_{\alpha}}=\left(\dot{\lambda}_{\text {con }}+\dot{\lambda}_{\text {dissip }}\right)\left(\lambda^{j(\alpha)}\right),
$$




$$
\frac{\psi^{j(\alpha+1)}-\psi^{j(\alpha)}}{\Delta t / N_{\alpha}}=\mathcal{L}_{\mathrm{FP}}\left(\psi^{j(\alpha)}, \lambda^{j(\alpha)}\right)
$$

where $\mathcal{L}_{\text {FP }}$ refers to the Fokker-Planck operator appearing on the right-hand side of Eq. (17). Then, set $\lambda^{j+1 / 2}=\lambda^{j\left(N_{\alpha}\right)}$ and $\psi^{j+1 / 2}=\psi^{j\left(N_{\alpha}\right)}$,

- Second half-time step

$$
\begin{aligned}
& \frac{\lambda^{j+1}-\lambda^{j+1 / 2}}{\Delta t}+\left(\mathbf{v}^{j} \cdot \nabla\right) \lambda^{j+1}=0 \\
& \frac{\psi^{j+1}-\psi^{j+1 / 2}}{\Delta t}+\left(\mathbf{v}^{j} \cdot \nabla\right) \psi^{j+1}=0 .
\end{aligned}
$$

The orientation tensor $\mathbf{S}^{j+1}$ may be calculated from

$$
\mathbf{S}^{j+1}(\mathbf{x}, t)=\int_{s=0}^{1} \int_{\theta=0}^{\pi} \int_{\varphi=0}^{2 \pi} \psi^{j+1}[\mathbf{x}, t, \mathbf{u}(\theta, \varphi), s] \mathbf{u}(\theta, \varphi) \mathbf{u}(\theta, \varphi) \sin \theta d \varphi d \theta d s,
$$

where $\mathbf{u}=\sin \theta \cos \varphi \mathbf{e}_{x}+\sin \theta \sin \varphi \mathbf{e}_{y}+\cos \theta \mathbf{e}_{z} \cdot \boldsymbol{\tau}^{j+1}$ then follows from Eq. (19) and the algorithm increments $j$ by 1 and returns to the Stokes system (21) and (22).

\section{B. Discretization in configurational space}

A discrete approximation to the configurational pdf $\psi$, expressing dependence on $s$ and on a generic point on the unit sphere in configurational space, may be written in the form

$$
\psi(\mathbf{u}, s, \mathbf{x}, t) \approx \sum_{i=0}^{1} \sum_{\ell=0}^{N_{s}} \sum_{n=0}^{N_{u}} \sum_{m=i}^{n} \sum_{i} \psi_{i, \ell, n, m}(\mathbf{x}, t) \Phi_{2 n, 2 m}^{i}(\theta, \varphi) L_{\ell}(s) .
$$

In Eq. (28) $\Phi_{n, m}^{i}=P_{n}^{m}(\cos \theta)[(1-i) \cos m \varphi+i \sin m \varphi](i=0,1)$ are spherical harmonics defined in terms of the associated Legendre polynomials $P_{n}^{m}$ and the spherical polar coordinates $\theta$ and $\varphi . L_{\ell}(s)$ is a degree $N_{s}$ polynomial, defined on $[0,1]$ by

$$
L_{\ell}(s)=h_{\ell}(\xi)
$$

where $\xi=2 s-1$ and

$$
h_{\ell}(\xi)=-\frac{1}{N_{s}\left(N_{s}+1\right) P_{N_{s}}\left(\xi_{\ell}\right)} \frac{\left(1-\xi^{2}\right) P_{N_{s}}^{\prime}(\xi)}{\left(\xi-\xi_{\ell}\right)}
$$

Here, $P_{N_{s}}(\xi)$ is the degree $N_{s}$ Legendre polynomial and the $\left\{\xi_{\ell}\right\}_{\ell=0}^{N}=0$ are the GaussLobatto-Legendre (GLL) points [see the book of Canuto et al. (1988), for example]. $h_{\ell}$ is the $\ell$ th Lagrange interpolating polynomial based on the GLL points and has the property that $h_{\ell}\left(\xi_{j}\right)=\delta_{\ell, j}, \ell, \ell=0, \ldots, N_{s}$. We note that only the spherical harmonics of even order appear in Eq. (28). This is because $\psi$ is an even function of $\mathbf{u}$.

Inserting Eq. (28) into the FP equation (17) for $\psi$ we now seek to simplify the terms in the square parentheses appearing on the right-hand side of Eq. (17). It is shown by Fan (1989a) that 


$$
\frac{\partial}{\partial \mathbf{u}} \cdot\left[(\mathbf{I}-\mathbf{u u}) \cdot \boldsymbol{\kappa} \cdot \mathbf{u} \Phi_{n, m}^{i}\right]=\sum_{k=m-2}^{m+2} \sum_{j=n-2}^{n+2} a_{n, j}^{m, k}\left[w_{j}^{k} \Phi_{j, k}^{i}+(-1)^{1-i} v_{j}^{k} \Phi_{j, k}^{1-i}\right]
$$

where the coefficients $a_{n, j}^{m, k}$ and the linear combinations of velocity gradients $w_{j}^{k}$ and $v_{j}^{k}$ are (helpfully) supplied by the same author [See Tables 1-3 of the paper by Fan (1989a)].

The diffusion term is much easier. The spherical harmonics are the eigenfunctions of the Laplace operator on the unit sphere; specifically,

$$
\frac{\partial}{\partial \mathbf{u}} \cdot \frac{\partial \Phi_{n, m}}{\partial \mathbf{u}}=-n(n+1) \Phi_{n, m}^{i}
$$

Using Eqs. (28) and (31) and (32), we form the product of Eq. (17) with a test function $\Phi_{2 p, 2 q}^{i}(\theta, \varphi) L_{k}(s)\left(i=0,1 ; p=0, \ldots, N_{u} ; q=i, \ldots, p ; k=0, \ldots, N_{s}\right)$ and integrate over configurational space $B(0,1) \times[0,1]$. The integral with respect to $s$ is evaluated using a Gauss-Lobatto quadrature rule, and orthogonality of the spherical harmonics over $B(0,1)$ is exploited. The time- and configurational space-discretized equations corresponding to Eqs. (24) and (26) now, therefore, become

$$
\begin{aligned}
& \frac{\omega_{k}}{\Delta t / N_{\alpha}}\left[\psi_{i, k, p, q}^{j(\alpha+1)}(\mathbf{x})-\psi_{i, k, p, q}^{j(\alpha)}(\mathbf{x})\right] \\
& \quad=-\omega_{k} \sum_{n=p-1}^{p+1} \sum_{m=q-1}^{q+1} a_{2 p, 2 n}^{2 q, 2 m}\left[w_{2 n}^{2 m} \psi_{i, k, n, m}^{j(\alpha)}(\mathbf{x})+(-1)^{i} v_{2 n}^{2 m} \psi_{1-i, k, n, m}^{j(\alpha)}(\mathbf{x})\right] \\
& \quad-\sum_{\ell=0}^{N_{s}} \psi_{i, \ell, p, q}^{j(\alpha)}(\mathbf{x})\left(\left[\dot{s}_{\mathrm{tot}} L_{l}(s)\right]^{\prime}, L_{k}(s)\right)_{N_{s}}-\frac{\dot{\lambda}_{\mathrm{dissip}}}{\lambda} \omega_{k} \psi_{i, k, q, p}^{j(\alpha)}(\mathbf{x}) \\
& \quad+\frac{1}{\pi^{2} \tau_{d}} \sum_{\ell=0}^{N_{s}} \psi_{i, \ell, p, q}^{j(\alpha)}\left(L_{\ell}^{\prime \prime}(s), L_{k}(s)\right)_{N_{s}}-2 p(2 p+1) \omega_{k} D \psi_{i, k, p, q}^{j(\alpha)},
\end{aligned}
$$

and

$$
\begin{aligned}
& \frac{1}{\Delta t}\left[\psi_{i, k, p, q}^{j+1}(\mathbf{x})-\psi_{i, k, p, q}^{j+1 / 2}(\mathbf{x})\right]+\left(\mathbf{v}^{j+1} \cdot \nabla\right) \psi_{i, k, p, q}^{j+1}(\mathbf{x})=0, \\
& i=0,1, \quad p=0, \ldots, N_{u}, \quad q=i, \ldots, p, \quad k=1, \ldots, N_{s}-1,
\end{aligned}
$$

with $i \leqslant m \leqslant n \leqslant N_{u}$ in the summation in the first term on the right-hand side of Eq. (33). $(\cdot, \cdot)_{N_{s}}$ denotes the $\left(N_{s}+1\right)$ point GLL quadrature evaluation of the $L^{2}$ inner product over $[0,1]$ :

$$
(u, v)_{N_{s}}=\sum_{k=0}^{N_{s}} \omega_{k} u\left(s_{k}\right) v\left(s_{k}\right)
$$

where $s_{k}=\left(\xi_{k}+1\right) / 2$ and $\omega_{k}$ are quadrature weights.

Once we have the pdf in the form (28), the components of the orientation tensor $\mathbf{S}$ $=\langle\mathbf{u u}\rangle$ may be computed from the formulas 


$$
\begin{gathered}
S_{x x}=4 \pi \sum_{l=0}^{N_{s}} \omega_{l}\left(\frac{1}{3} \psi_{0, \ell, 0,0}-\frac{1}{15} \psi_{0, \ell, 1,0}+\frac{2}{5} \psi_{0, \ell, 1,1}\right), \\
S_{y y}=4 \pi \sum_{l=0}^{N_{s}} \omega_{l}\left(\frac{1}{3} \psi_{0, \ell, 0,0}-\frac{1}{15} \psi_{0, \ell, 1,0}-\frac{2}{5} \psi_{0, \ell, 1,1}\right), \\
S_{z z}=4 \pi \sum_{l=0}^{N_{s}} \omega_{l}\left(\frac{1}{3} \psi_{0, \ell, 0,0}+\frac{2}{15} \psi_{0, \ell, 1,0}\right), \\
S_{x y}=\frac{8 \pi}{5} \sum_{l=0}^{N_{s}} \omega_{l} \psi_{1, \ell, 1,1},
\end{gathered}
$$

the other components being zero in a flow parallel to the $x y$ plane.

\section{Discretization in physical space}

Finally, we discretize Eqs. (21), (22), (25), and (26) in physical space using a spectral element method. This is done by choosing tensorized bases in real space for all dependent variables-with the exception of the pressure-consisting of polynomials of degree $N$ in each spatial variable. The pressure basis polynomials are chosen to be of degree 2 less in each direction so as to ensure that the discrete problem is well posed [see Maday et al. (1992)]. A discrete system of equations is set up using the Galerkin method for Eqs. (21) and (22) and an Uzawa algorithm (block Gaussian elimination) is used to construct a discrete Poisson-type problem for the pressure from Eqs. (21) and (22). For details of the implementation of the Uzawa algorithm in the viscoelastic context the reader is referred to the paper of Owens and Phillips (1996), for example.

Equations (23) and (24) are solved separately at each collocation point. An SUPG element-by-element spectral element method with constant upwinding factor, as detailed by Chauvière and Owens (2001), is used to treat convection Eqs. (25) and (26).

\section{RESULTS}

\section{A. Homogeneous flows of the UÖ model: Stochastic simulations [Fang et al. (2000)] versus the Fokker-Planck method}

The predictions of the UÖ model for homogenous shear and extensional flows are described in detail by Fang et al. (2000). Here, we want to validate our numerical method by comparing the results with those from the stochastic simulations of Fang et al. We also want to investigate the efficiency of the two approaches. All CPU times quoted in Sec. IV A are for a Pentium III $800 \mathrm{MHz}$ machine and the parameters of the UÖ model are taken to be $\lambda_{\max }=21$ and $\tau_{d} / \tau_{s}=50$.

\section{Startup shear flow}

We report here the results for homogeneous startup shear flow. That is, we solve the FP equation (17) coupled with Eq. (16) with the steady velocity field

$$
\mathbf{v}=\left(v_{x}, v_{y}, v_{z}\right)=(\dot{\gamma} y, 0,0) \text {. }
$$

Figure 1 shows the relative errors for $\tau_{x y}$ after reaching the steady state with the FP-based method described in Sec. III for three different values of the dimensionless shear rate $\dot{\gamma} \tau_{d}=\{3,80,300\}$, taking the value computed on a finer grid $\left(N_{u}=N_{s}\right.$ $=40$ ) as the exact one. Each data point in Fig. 1 is labeled first with the CPU time 


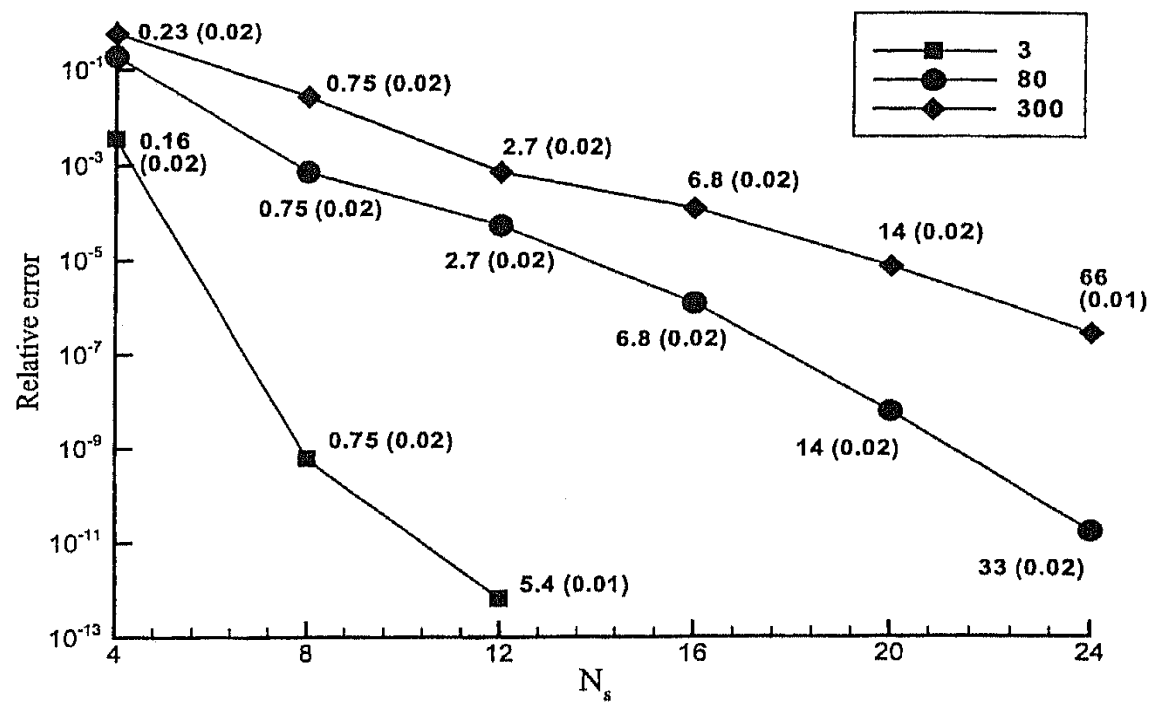

FIG. 1. Relative error for $\tau_{x y}$ for the simple shear flow (35) at three values of $\dot{\gamma} \tau_{d}$ using $N_{u}=N_{s}=4,8,12$, 16,20 , and 24. Each data point is labeled with the total CPU time in seconds and, in parentheses, the value of $\dot{\gamma} \Delta t$ used. Final time $=100 / \dot{\gamma}$.

required to reach a final time of $100 / \dot{\gamma}$, and second in parentheses, we indicate the dimensionless time step $\dot{\gamma} \Delta t$ chosen. The choice of the time step was made by performing a run at $\dot{\gamma} \Delta t=0.02$ and (if necessary) dividing this successively by $2,4, \ldots$, etc., until convergence was achieved. We see that the scheme manifests exponential convergence. However, as is to be expected, the rate of convergence gets lower with increasing $\dot{\gamma} \tau_{d}$

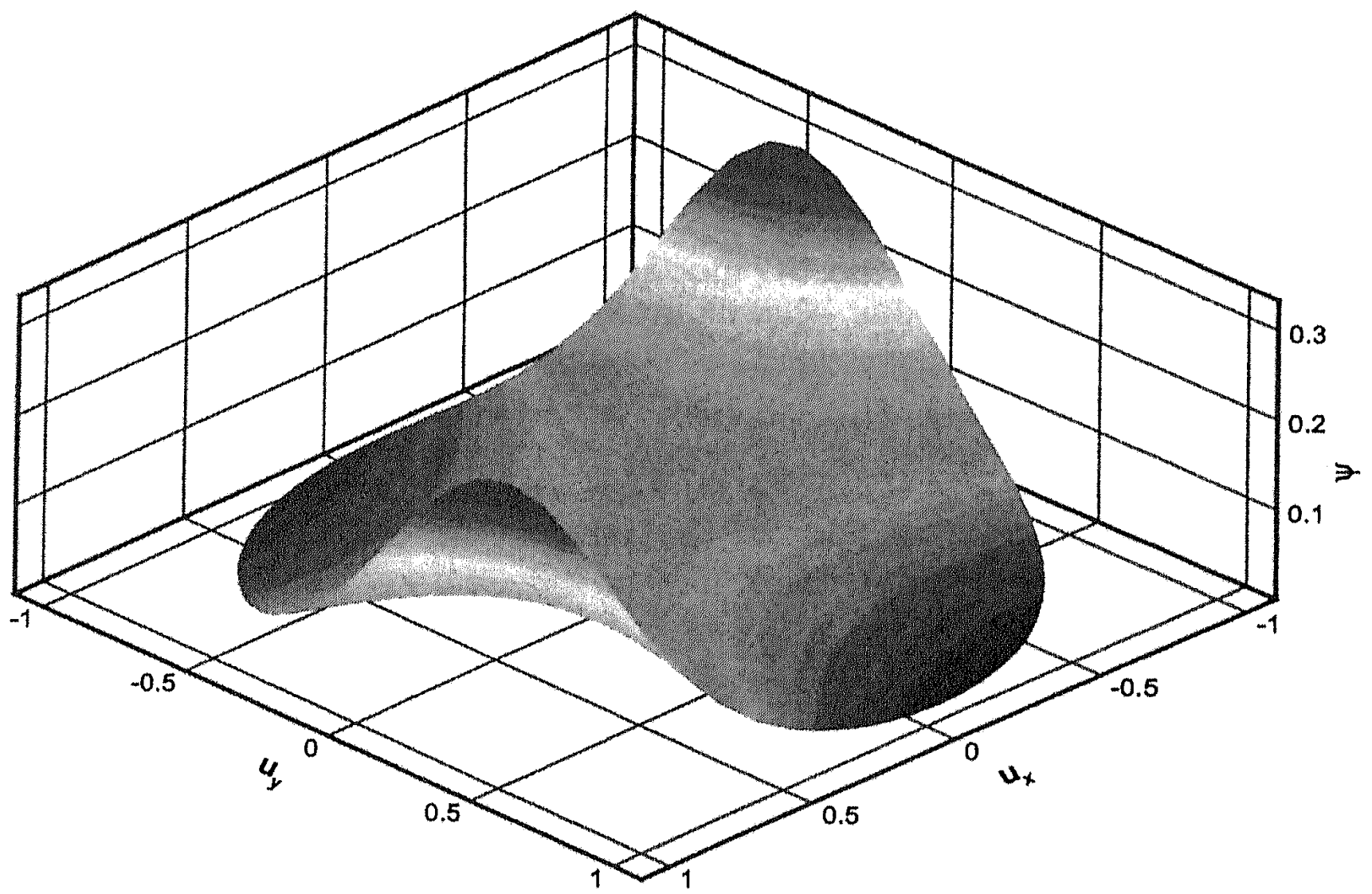

FIG. 2. Pdf $\psi$ as a function of $u_{x}$ and $u_{y}$ at $s=0.5$ for the simple shear flow (35) at $\dot{\gamma} \tau_{d}=3$ eomputed with $N_{u}=N_{s}=12$. 


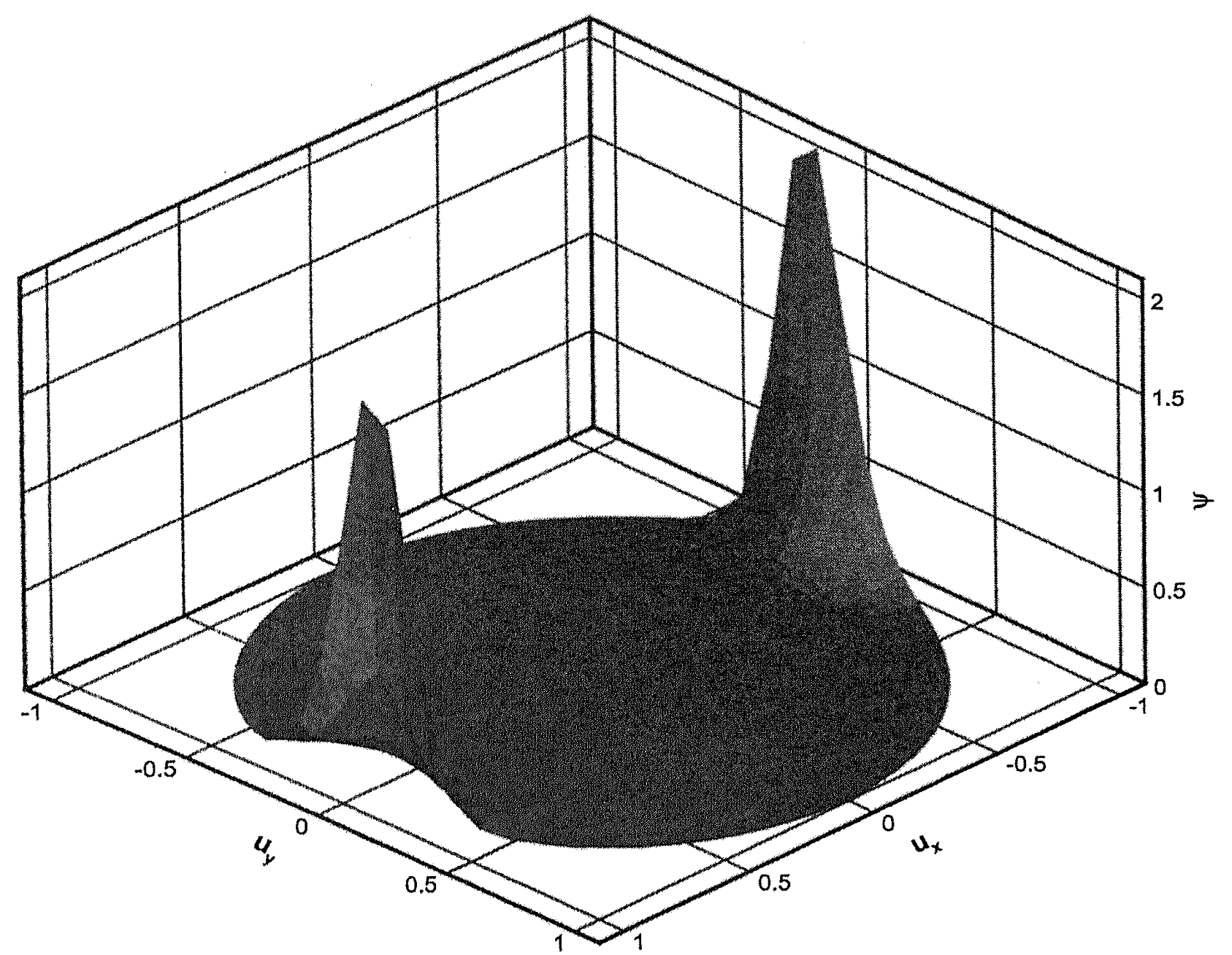

FIG. 3. Pdf $\psi$ as a function of $u_{x}$ and $u_{y}$ at $s=0.5$ for the simple shear flow (35) at $\dot{\gamma} \tau_{d}=800$ computed with $N_{\mu}=N_{s}=16$.

The reason for this becomes clear if we compare the solutions for the pdf $\psi$ at the shear rates $\dot{\gamma} \tau_{d}=3$ and 800 shown in Figs. 2 and 3, respectively, where $\psi$ is plotted as a function of $u_{x}$ and $u_{y}$ at $s=0.5$ for the steady state, i.e., $\psi$ evaluated at $s=0.5$ on the upper hemisphere pointed to by $\mathbf{u}$ is projected onto the unit disk. We see that the solution in Fig. 2 is very smooth, but that that in Fig. 3 has two spikes that can be captured accurately only on a sufficiently refined mesh.

Not surprisingly, our FP method is particularly advantageous in comparison with the stochastic simulations of Fang et al. (2000) at low shear rates. We compare the respective

TABLE 1. Convergence of our FP method for the simple shear flow (35) at $\dot{\gamma} \tau_{d}=3$. Final time $100 / \dot{\gamma}$

\begin{tabular}{lccccc}
\hline \hline$N_{u}$ & $N_{s}$ & $\dot{\gamma} \Delta t$ & $\tau_{x y}$ & $\left|\Delta \tau_{x y} / \tau_{x y}\right|$ & CPU time (seconds) \\
\hline 4 & 4 & 0.02 & 0.509716873089292 & $3.6 \times 10^{-3}$ & 0.16 \\
8 & 8 & 0.02 & 0.511583481828495 & $6.0 \times 10^{-10}$ & 0.75 \\
12 & 12 & 0.01 & 0.511583481521587 & $6.5 \times 10^{-13}$ & 5.4 \\
16 & 16 & 10.005 & 0.5115834815210698 & $3.6 \times 10^{-13}$ & 27 \\
20 & 20 & 0.0025 & 0.511583481521067 & $3.6 \times 10^{-13}$ & 113 \\
24 & 24 & 0.00125 & 0.511583481521072 & $3.5 \times 10^{-13}$ & 527 \\
28 & 28 & 0.000625 & 0.511583481521099 & $3.0 \times 10^{-13}$ & 2535 \\
40 & 40 & $1.5625 \times 10^{-4}$ & 0.511583481521253 & - & 34161 \\
\hline
\end{tabular}


TABLE II. Convergence of the stochastic simulations of Fan et al. (2000) for the simple shear flow (35) at $\dot{\gamma} \tau_{d}=3$. Final time $100 / \dot{\gamma}$.

\begin{tabular}{lcccccc}
\hline \hline$N_{\text {sample }}{ }^{\mathrm{a}}$ & $N_{\text {block }}$ & $\dot{\gamma} \Delta t$ & $\tau_{x y}$ & $\delta \tau_{x y}$ & $\left|\Delta \tau_{x y} / \tau_{x y}\right|$ & CPU time (seconds) \\
\hline 10000 & 10 & 0.04 & 0.5073 & $2.7 \times 10^{-3}$ & $8.4 \times 10^{-3}$ & $6(4)$ \\
10000 & 10 & 0.02 & 0.5097 & $4.3 \times 10^{-3}$ & $3.6 \times 10^{-3}$ & 1166 \\
10000 & 10 & 0.01 & 0.5103 & $3.6 \times 10^{-3}$ & $2.4 \times 10^{-3}$ & 2320 \\
\hline 10000 & 100 & 0.04 & 0.5074 & $8.7 \times 10^{-4}$ & $8.1 \times 10^{-3}$ & 6054 \\
10000 & 100 & 0.02 & 0.5091 & $1.4 \times 10^{-3}$ & $4.8 \times 10^{-3}$ & 11672 \\
10000 & 100 & 0.01 & 0.5104 & $1.2 \times 10^{-3}$ & $2.3 \times 10^{-3}$ & 23225 \\
\hline \hline
\end{tabular}

an the work of Fang et al. the simulations were performed in $N_{\text {block }}$ independent blocks, in each of which $N_{\text {sample }}$ trajectories of the stochastic processes $\mathbf{u}$ and $s$ were allowed to propagate. See Fang et al. (2000).

CPU times at $\dot{\gamma} \tau_{d}=3$ in Tables I and II. In Table I we show the computed values of $\tau_{x y}$, the absolute relative errors $\left|\Delta \tau_{x y} / \tau_{x y}\right|$ (assuming the solution computed with $N_{u}$ $=N_{s}=40$ to be exact), and the CPU times for our method. In Table II we supply the

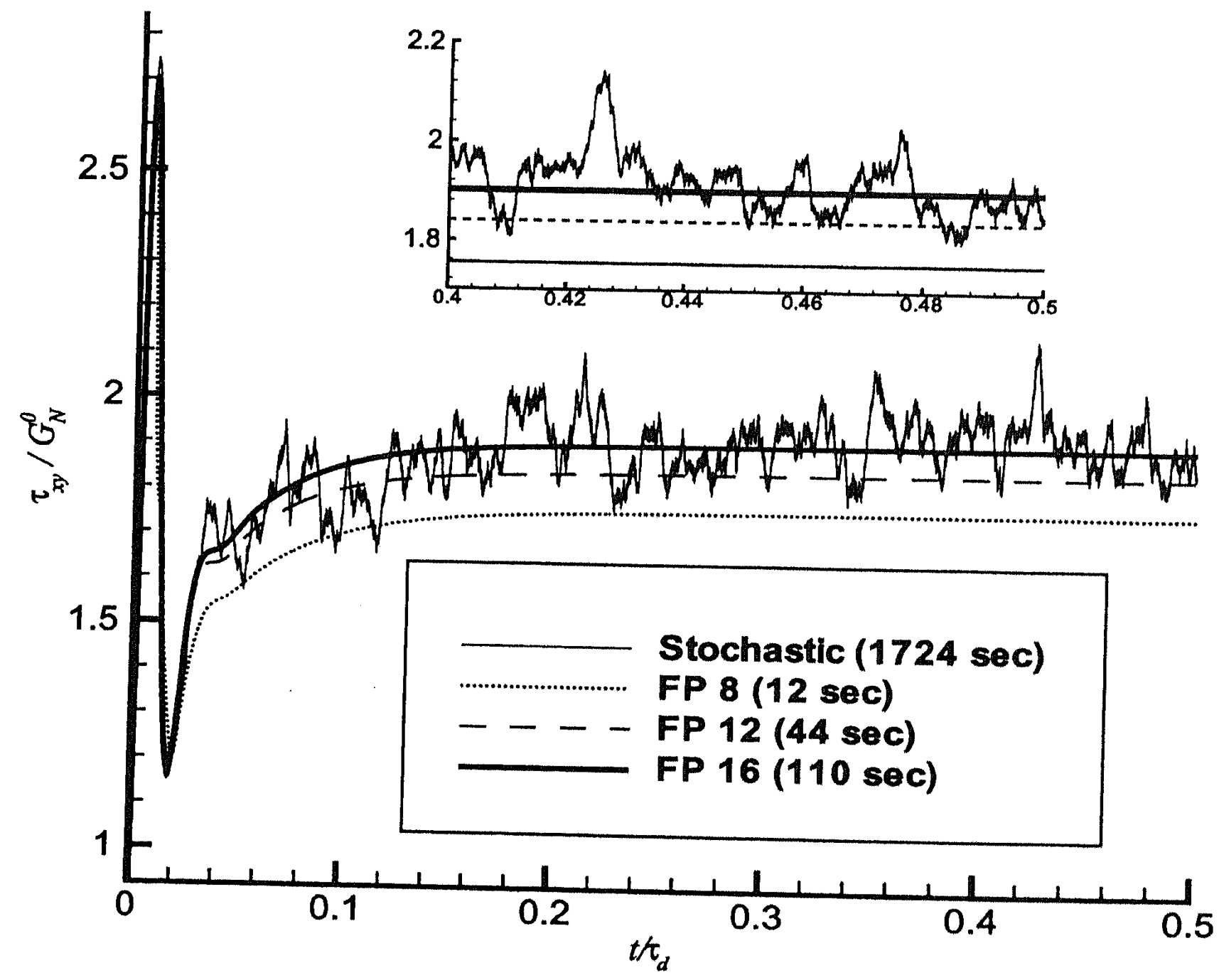

FIG. 4. Evolution of $\tau_{x y}$ for the startup simple shear flow (35) at $\dot{\gamma} \tau_{d}=800$. FP $M$ stands for the FP. based numerical method with $N_{u}=N_{s}=M$ and $\Delta t=0.005 / \dot{\gamma}$. The stochastic simulation was implemented with $N_{\text {sample }}=10000, N_{\text {block }}=2$, and $\Delta t=0.01 / \dot{\gamma}$. The CPU times on a Pentium III 800 MHz machine are also
indicated. 


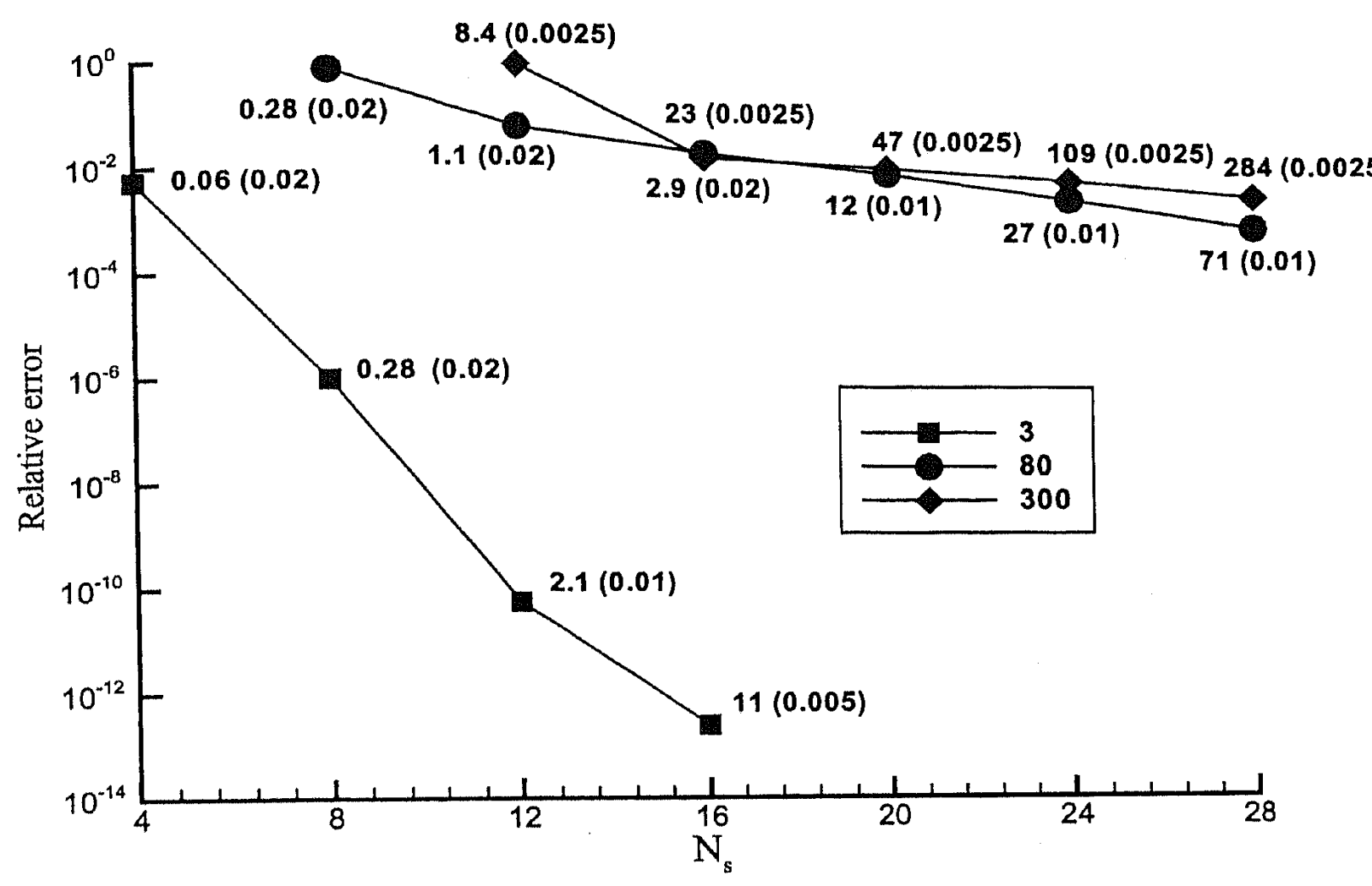

FIG. 5. Relative error for $N_{1}$ for the uniaxial extensional flow (36) at three values of $\dot{\epsilon} \tau_{d}$ using $N_{u}=N_{s}$ $=4,8,12,16,20,24$ and 28 . Each data point is labeled with the total CPU time in seconds and, in parentheses, the value of $\dot{\epsilon} \Delta t$ used. Final time $=50 / \dot{\epsilon}$.

values of $\tau_{x y}$ computed with the method of Fang et al. (2000) and averaged over the final 200 time steps, the standard deviations $\delta \tau_{x y}$ over the same time interval, the absolute relative errors $\left|\Delta \tau_{x y} / \tau_{x y}\right|$ (again, assuming that our FP solution computed with $N_{u}$ $=N_{s}=40$ is exact), and the CPU times. We see that both methods give approximately the same results, but that our FP method can be thousands of times faster for the same level of accuracy. As an example, in the FP shear flow calculation with $N_{u}=N_{s}=4$ detailed in Table I, only 0.16 CPU seconds were taken to compute a solution having a relative error in the shear stress $\tau_{x y}$ of $3.6 \times 10^{-3}$. The vast majority of this time $(99.9 \%)$ was consumed performing the iterations (23) and (24) and the remaining time mainly with the construction of the coefficient matrix for the FP operator in Eq. (24). For the same level of accuracy it may be seen from Table II that 1166 CPU seconds were required by the Fang et al. stochastic algorithm to reach the same final time of $100 / \dot{\gamma}$. The reason for the significant difference in CPU time between the two methods is that reduction of the variance in the stochastic algorithm requires that $N_{\text {sample }} \times N_{\text {block }}$ be chosen sufficiently large and accuracy demands that $\Delta t$ be chosen sufficiently small.

What is less evident is that our method can be more efficient than the stochastic simulation technique even at the high shear rate $\dot{\gamma} \tau_{d}=800$, as may be seen from Fig. 4 . Although relatively high values for $N_{u}$ and $N_{s}$ are needed in order to match the "deterministic" solution with the stochastic one, our method gives about a 15-fold gain in CPU time.

\section{Uniaxial extensional flow}

We report here the results for homogeneous startup uniaxial extensional flow with a velocity field of the form 


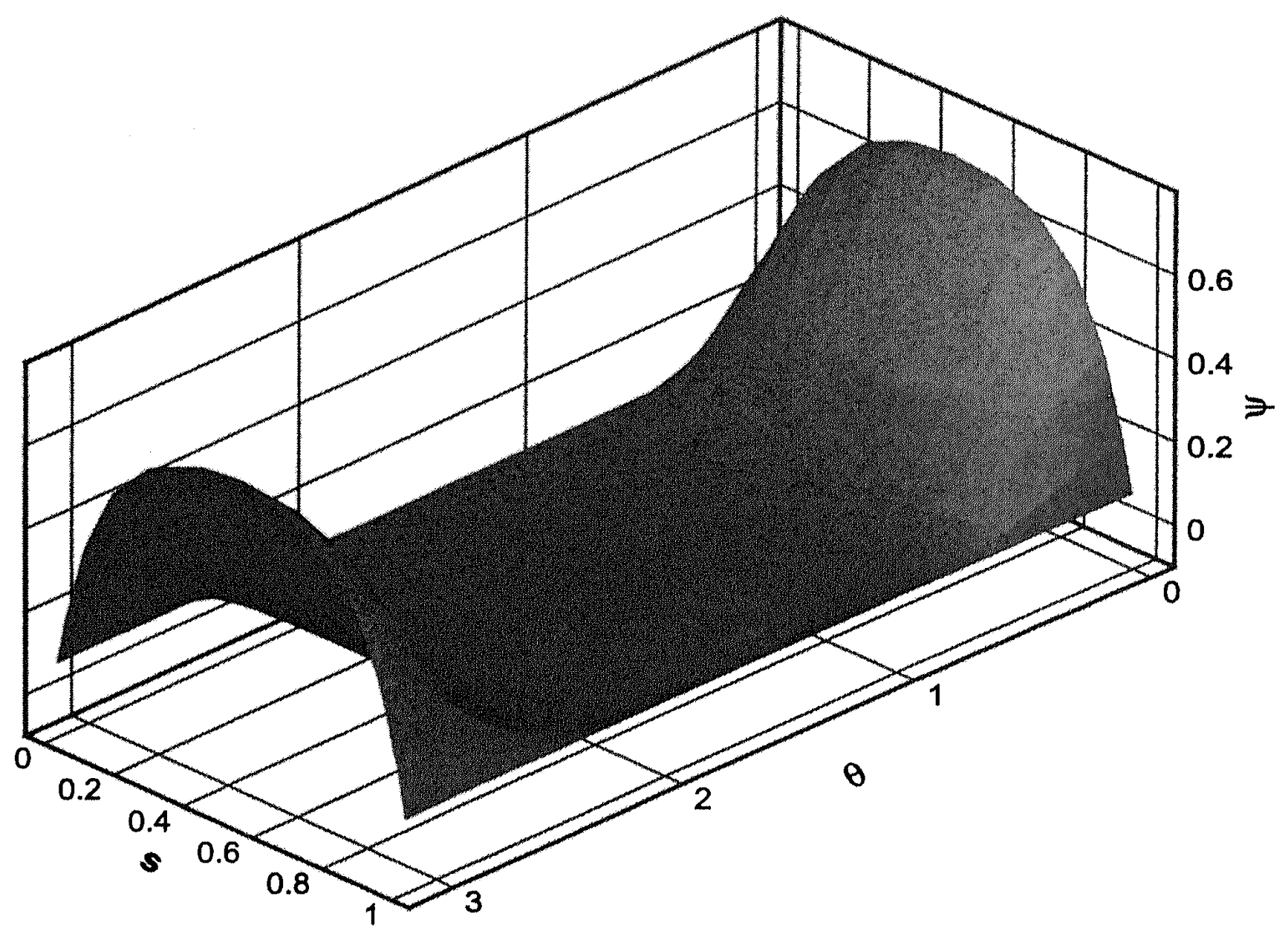

FIG. 6. Pdf $\psi$ for the uniaxial extensional flow (36) at $\dot{\epsilon} \tau_{d}=3$ computed with $N_{u}=N_{s}=12$.

$$
\mathbf{v}=\left(v_{x}, v_{y}, v_{z}\right)=(-\dot{\epsilon} x / 2,-\dot{\epsilon} y / 2, \dot{\epsilon} z)
$$

Figure 5 shows the relative errors for the normal stress difference $N_{1}=\tau_{z z}-\tau_{x x x}$ after reaching the steady state (final time 50/ $\dot{\epsilon}$ ) with the FP-based numerical method for three different values of the dimensionless extensional rate $\dot{\epsilon} \tau_{d}=\{3,80,300\}$. "Exact" solutions for these three extensional rates were calculated with $N_{u}=N_{s}=40$ ). In a similar manner to Fig. 1, each data point in Fig. 5 is labeled first with the CPU time required to reach a final time of $50 / \dot{\epsilon}$ and then, in parentheses, the dimensionless time step $\dot{\epsilon} \Delta t$ chosen.

The scheme again converges exponentially fast but, of course, the convergence rate decreases with increasing $\dot{\epsilon} \tau_{d}$. We are able to demonstrate even more clearly than in the previous subsection why this should be so. $\psi$ is plotted in Figs. 6 and 7 for $\dot{\boldsymbol{\epsilon}} \tau_{d}=3$ and 300 as a function of $\theta$ and $s$ (the solution for $\psi$ does not depend on $\varphi$ in this extensional flow since the velocity field is invariant to rotation about the $z$ axis). We see that the solution at $\dot{\boldsymbol{\epsilon}} \tau_{d}=300$ has sharp boundary layers at $s=0$ and 1 , which a mesh of $N_{u}$ $=N_{s}=12$ is unable to resolve adequately; numerical oscillations being the result. Increasing the resolution in configurational space to $N_{u}=N_{s}=50$ captures the boundary layers satisfactorily, as shown in Fig. 8. However, our method is at least as efficient as the stochastic simulation for the same accuracy level as may be seen from Fig. 9. Reasonable agreement is found in the steady state values of $N_{1}$ at $\dot{\epsilon} \tau_{d}=300$ predicted by run "c" of the FP method and run "f" of the stochastic method of Fang et al. (2000). Having said this, whereas run "c" required just $437 \mathrm{CPU}$ seconds, the stochastic calcu- 


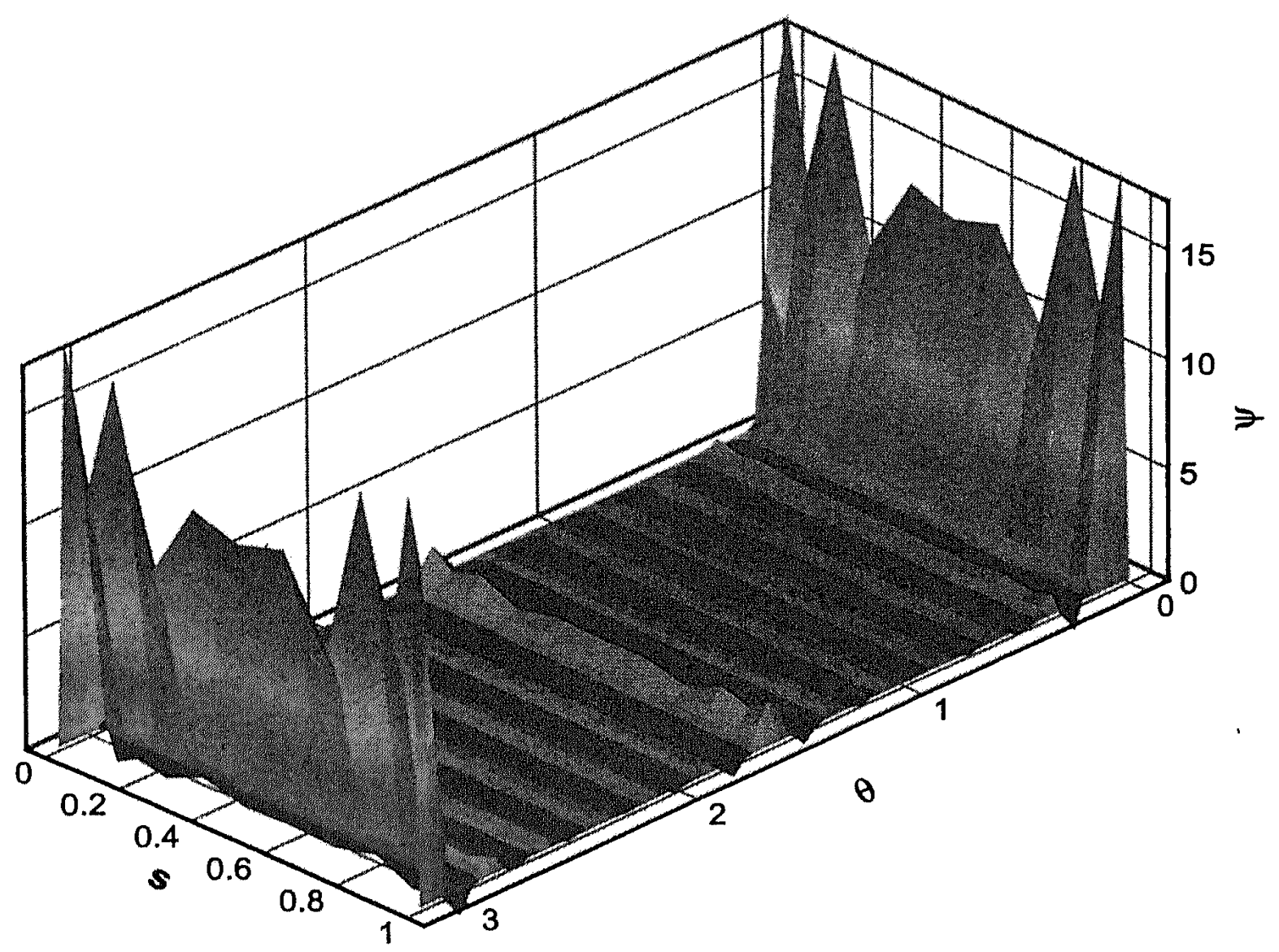

FIG. 7. Pdf $\psi$ for uniaxial extensional flow (36) at $\dot{\epsilon} \tau_{d}=300$ computed with $N_{u}=N_{s}=12$.

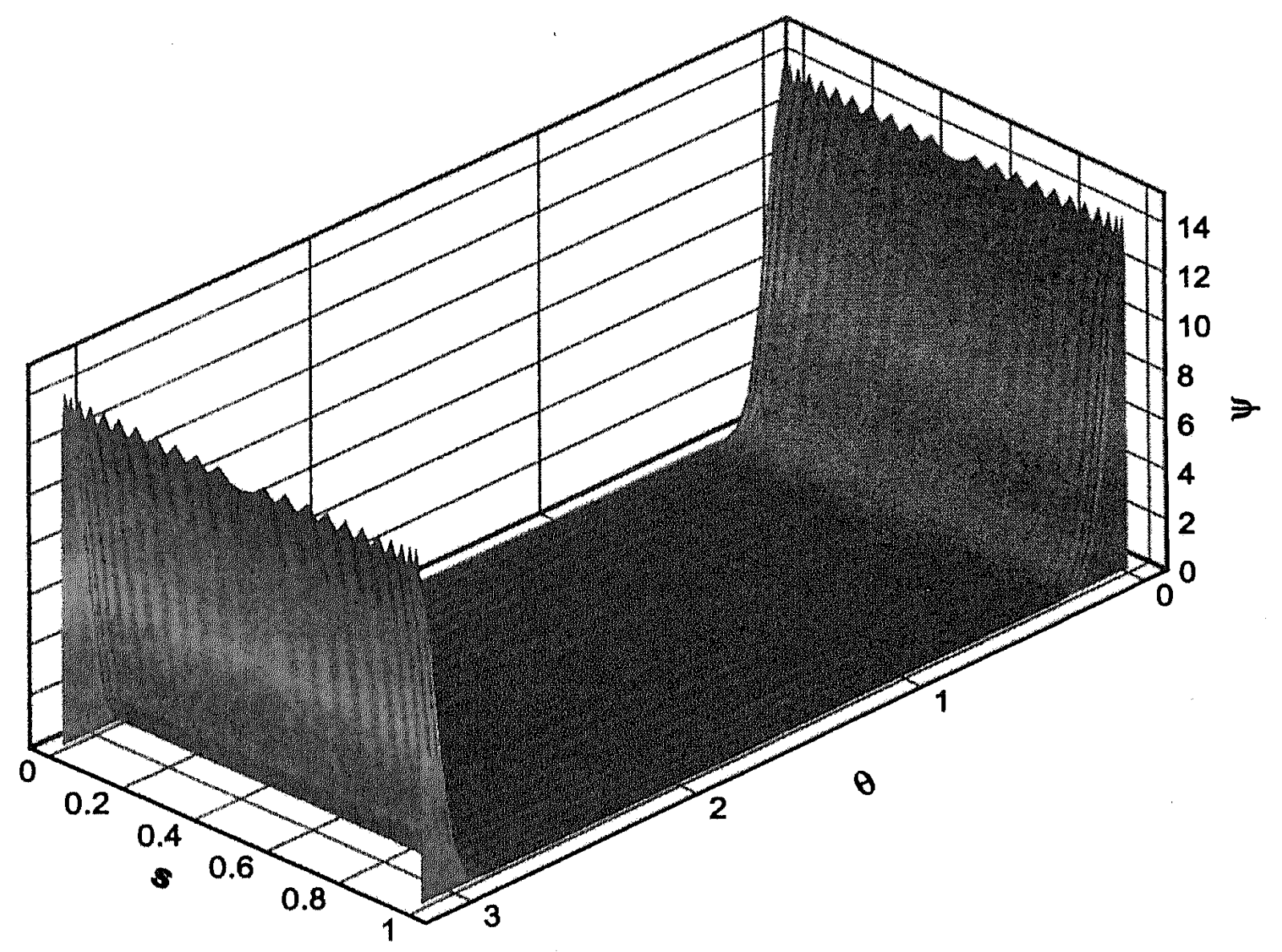

FIG. 8. Pdf $\psi$ for uniaxial extensional flow (36) at $\dot{\epsilon} \tau_{d}=300$ computed with $N_{u}=N_{s}=50$. 


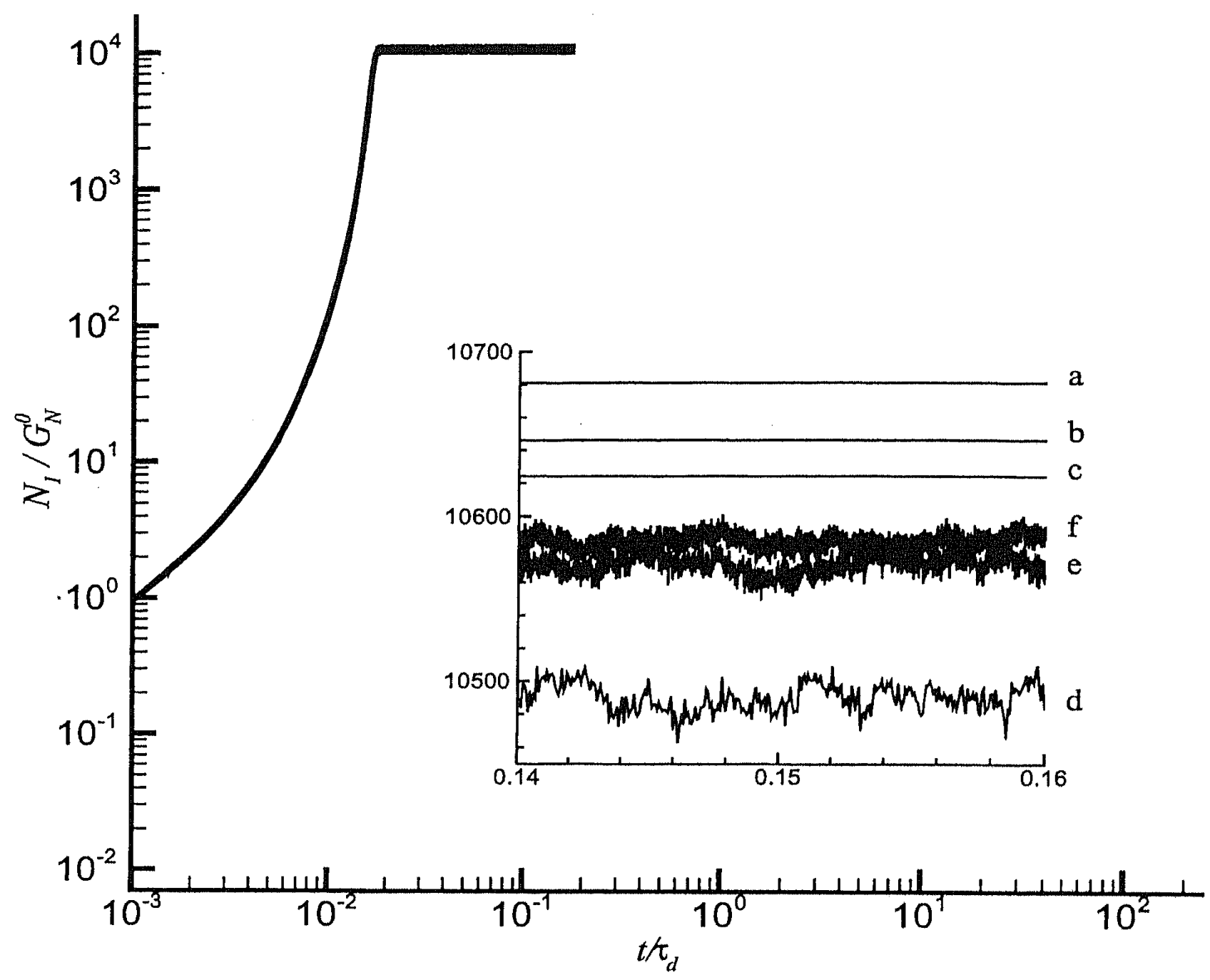

FIG. 9. Evolution of $N_{1}$ for the startup uniaxial extensional flow (36) at $\dot{\epsilon} \tau_{d l}=30$ ) with a $z 00 m$ on steady state regime. (a) FP-based simulation with $N_{u}=N_{s}=24, \Delta t=0.0025 / \dot{\epsilon}$, CPU time 1(1) seconds; (b) the same with $N_{u}=N_{s}=28$, CPU time 284 seconds; (c) the same with $N_{u}=N_{s}=32$, CPU time 437 seconds; (d) stochastic simulation with $N_{\text {sample }}=10000, N_{\text {block }}=2, \Delta t=0.02 / \dot{\epsilon}$, CPU time 107 seconds; (e) the same with $\Delta t=0.002 / \dot{\epsilon}$, CPU time 1063 seconds; and (f) the same with $\Delta t=0.0002 / \dot{\epsilon}$. CPU time $10609 \mathrm{~s}$. All CPU times quoted are for a Pentium III $800 \mathrm{MHz}$ machine.

lation took 10609 seconds using the same hardware. Although the convergence of both the FP and stochastic approaches is rather slow in the case of large extensional rates, the CPU time is always less for our method than for the stochastic one.

To facilitate comparisons of the computational cost for different levels of configurational mesh resolution for both the startup shear flow (35) and the uniaxial extensional flow (36), we have computed the CPU time per time step for each of the values of $N_{u}$ $=N_{s}$ selected in Figs. 1 and 5. These results are presented in graphical form in Fig. 10 on a $\log -\log$ scale and indicate that the CPU requirement increases in both cases no more rapidly than $O\left(N_{s}^{4}\right)$.

\section{B. Two-dimensional flow past a confined cylinder}

Having validated our numerical method for startup shear flow and extensional flow in Secs. IV A 1 and IV A 2, we now wish to evaluate, for the first time, the UÖ model in a complex flow. For this we choose the benchmark problem of flow past a confined cylinder (see Fig. 11) with a cylinder radius to half-channel ratio of $R / H=0.5$. The cylinder 


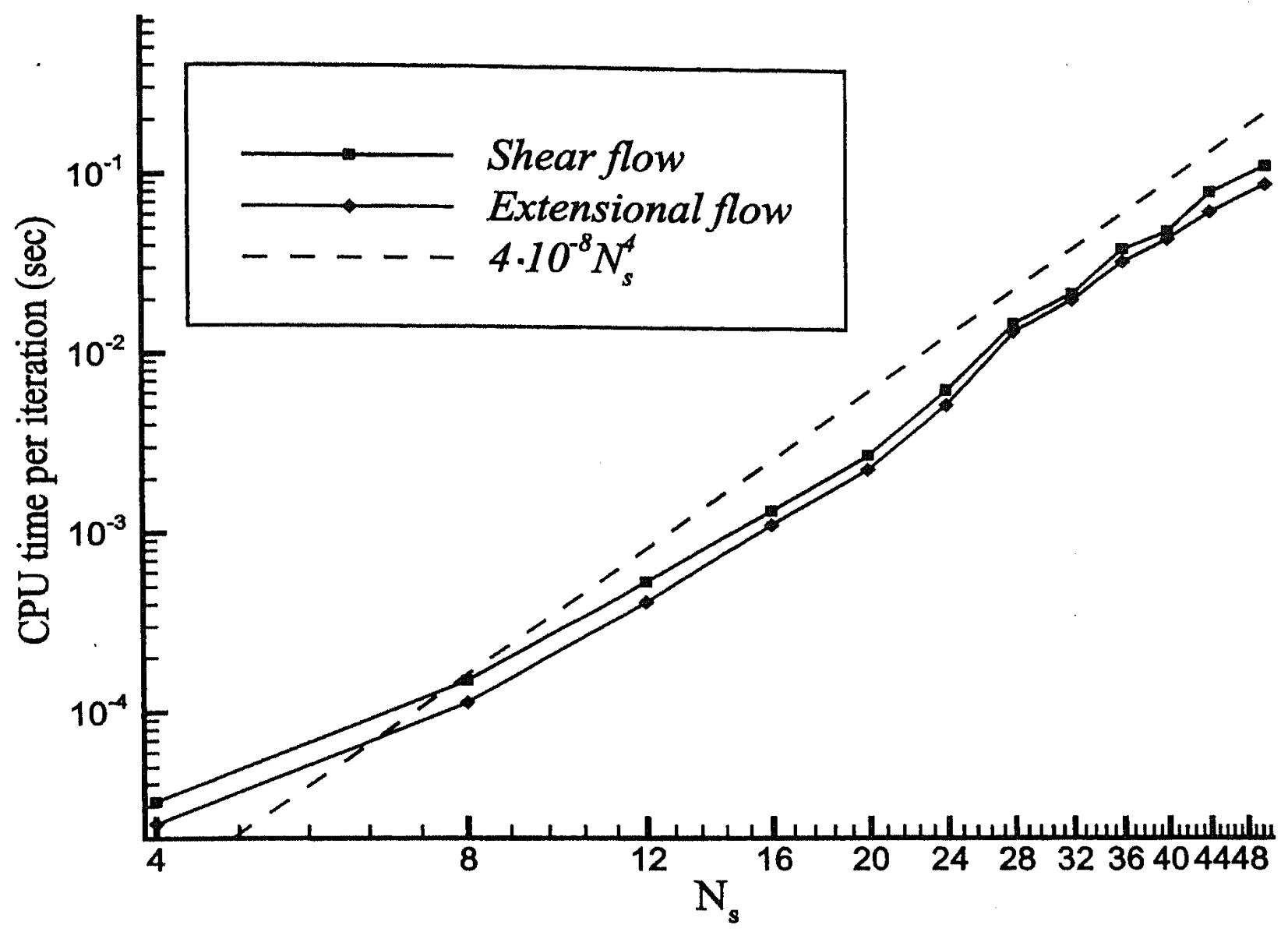

FIG. 10. CPU time (seconds) per time step for the simple shear flow (35) and the startup uniaxial extensional flow (36) vs the configurational resolution $N_{s}$.

radius $R$ is chosen as the characteristic length scale with respect to which the spatial variables are nondimensionalized [see Eq. (20)] and a Deborah number De for this flow is defined as

$$
\mathrm{De}=\bar{U}^{*} \equiv \frac{\bar{U} \tau_{d}}{R}
$$

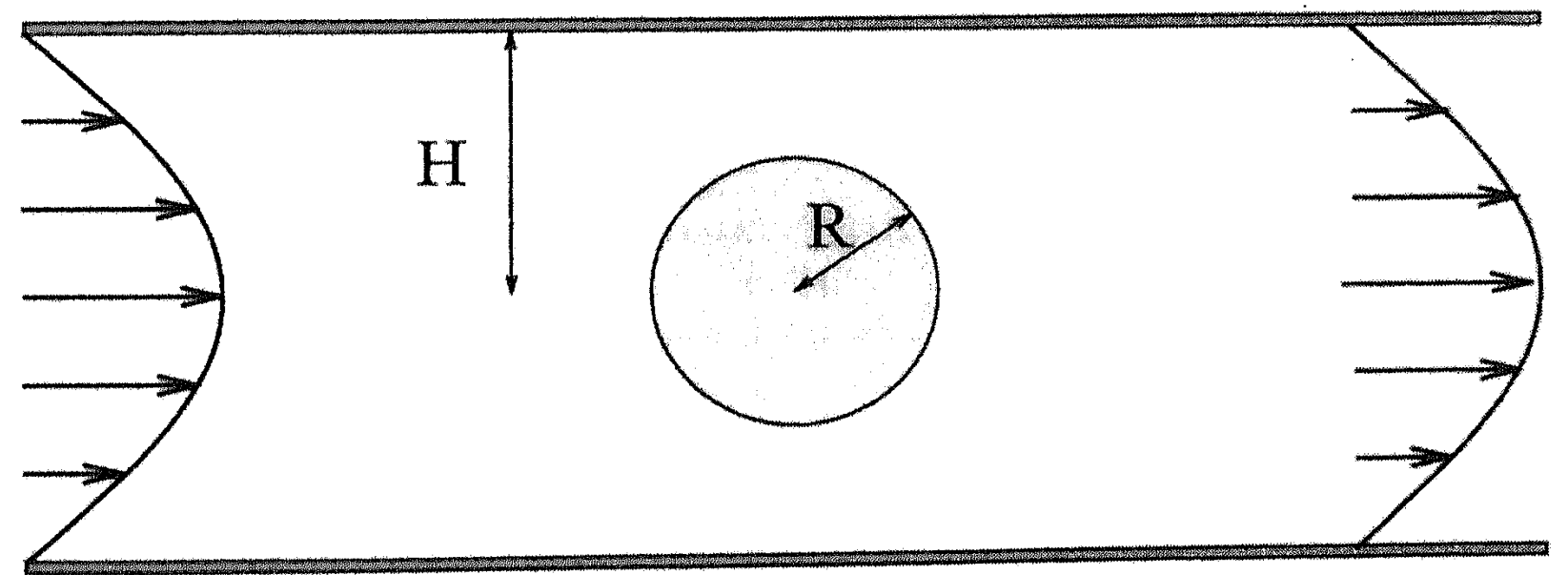

FIG. 11. Flow past a confined cylinder: Schematic of flow geometry. The cylinder has radius $R$ and the half-channel width is $H$. 


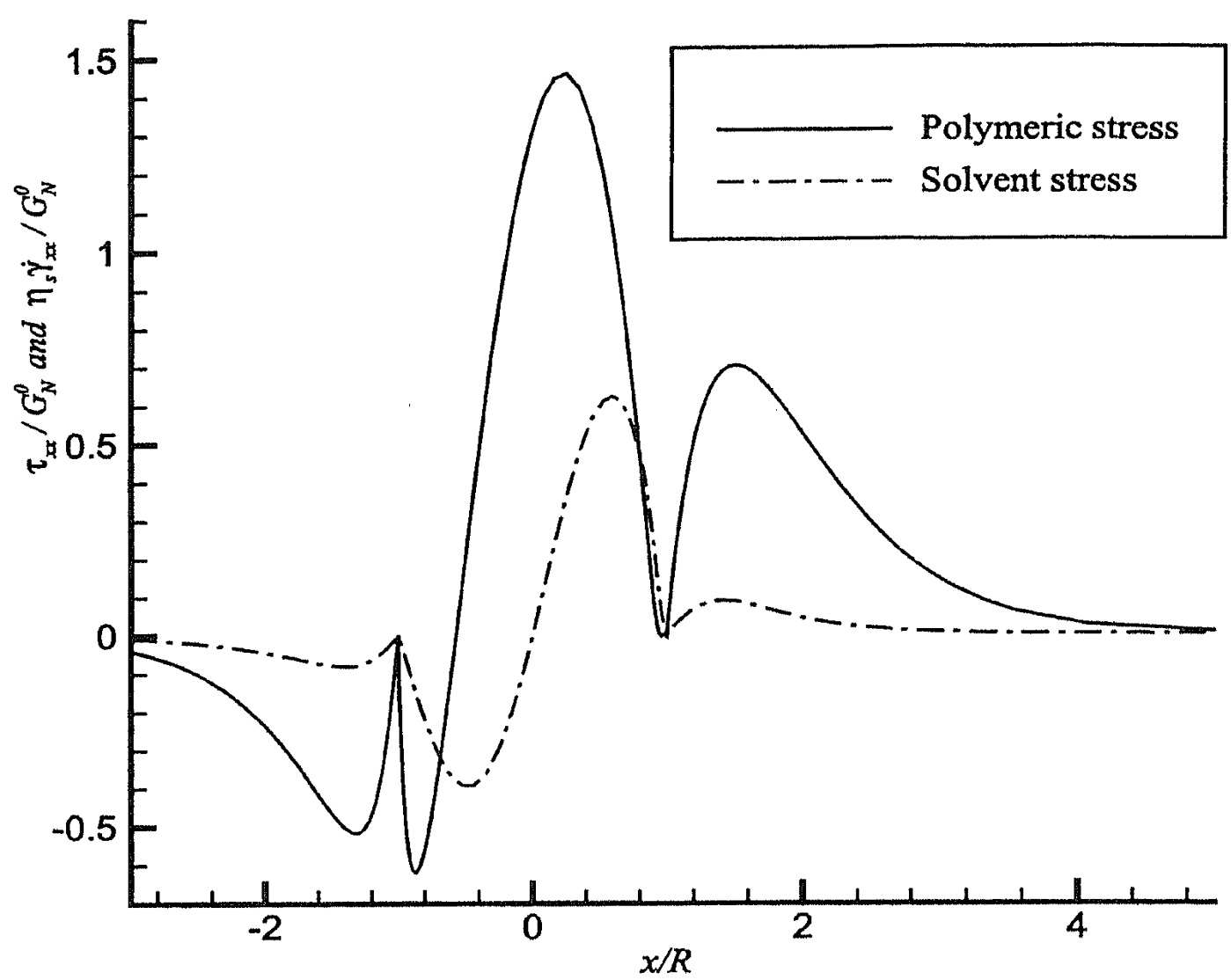

FIG. 12. Flow past a confined cylinder: Non-dimensionalized solvent $\left(\eta_{s} \dot{\gamma}_{s, x} / G_{N}^{()}\right)$and elastic $\left(\tau_{x, 1} / C_{N}^{()}\right)$ contributions to the $x x$ component of the Cauchy stress tensor along the axis of symmetry and on the cylinder surface $\left(-1 \leqslant x / R \leqslant 1\right.$ ) for $U \ddot{O}$ model at De $=0.6 . \eta_{s} / G_{N}^{(0} \tau_{d}=0 .\left(15, \beta=0, N_{1}=N_{s}=14\right.$, and $N=10$.

where $\vec{U}$ is the mean velocity in the inflow/outflow section of the channel. For all the calculations presented here we have chosen $\lambda_{\max }=21$ and $\tau_{d} / \tau_{s}=50$. Unless otherwise indicated in a figure caption, the discretization in configurational space was chosen as $N_{u}=N_{s}=14$. The flow domain was decomposed into 30 spectral elements and, unless indicated otherwise, degree $N=10$ polynomials in both spatial directions were used for the representation of all dependent variables with the exception of the pressure. Upstream and downstream channel lengths of 50 cylinder radii were chosen and periodic boundary conditions for all the field variables except the pressure were applied (the pressure is periodic up to addition by a linear function).

\section{Comparison of UÖ model with DE and MLD models [Peters et al. (2000b)] at low Deborah numbers}

Direct comparisons of the UÖ results with those obtained for the single-mode DE model [Doi and Edwards (1978a, 1978b, 1978c)] and the MLD model [Mead et al. (1998)] over long time intervals are not possible using our FP method-in neither case is there a diffusion term in $\mathbf{u}$ in the FP equation, provoking numerical instabilities at modest Deborah numbers. However, we are able to compare our results at De $=0.3$ and 0.6 with those obtained for these two models by Peters et $a l$. $(200)(\mathrm{b})$, who used a deformation field method [Peters et al. (2000a)] and the same value as above for $\tau_{d l} / \tau_{s}$.

Throughout this subsection, for both our results and those of Peters ' 1 al. "the dimensionless solvent viscosity $\eta_{s}^{*}$ is set equal to 0.05. At a Deborah number of 0.6 , the viscous stresses play an important but not yet dominant role, as may be seen from Fig. 12 , where both the elastic and viscous contributions to the $x x$ component of the Cauchy 


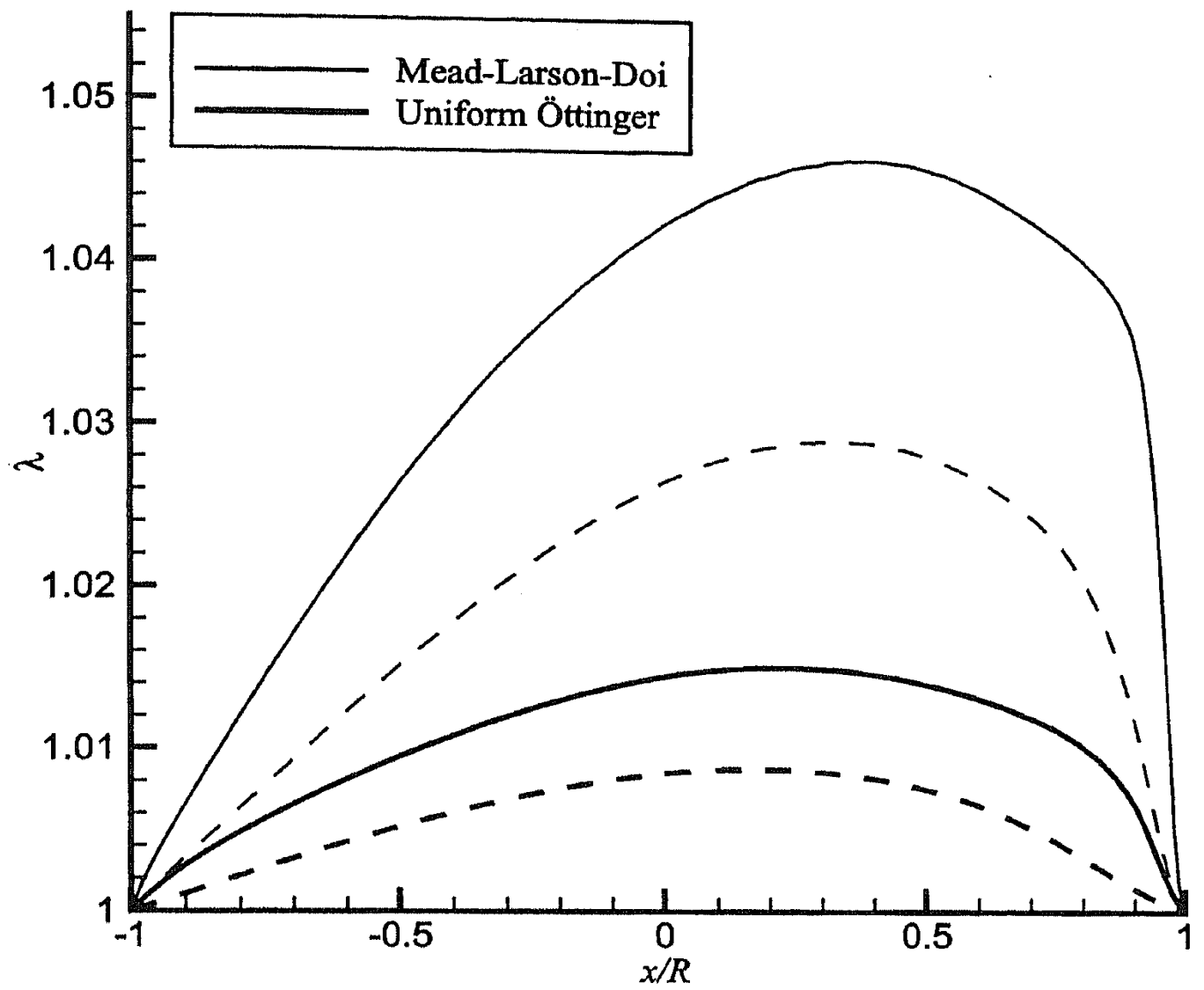

FIG. 13. Flow past a confined cylinder: Comparison of stretch parameter $\lambda$ along the cylinder wall for MLD model [Peters at al. $(20(0) \mathrm{b})$ ] and UÖ model. Dashed curves: De $=0.3$. Solid curves: $\mathrm{De}=0.6$.

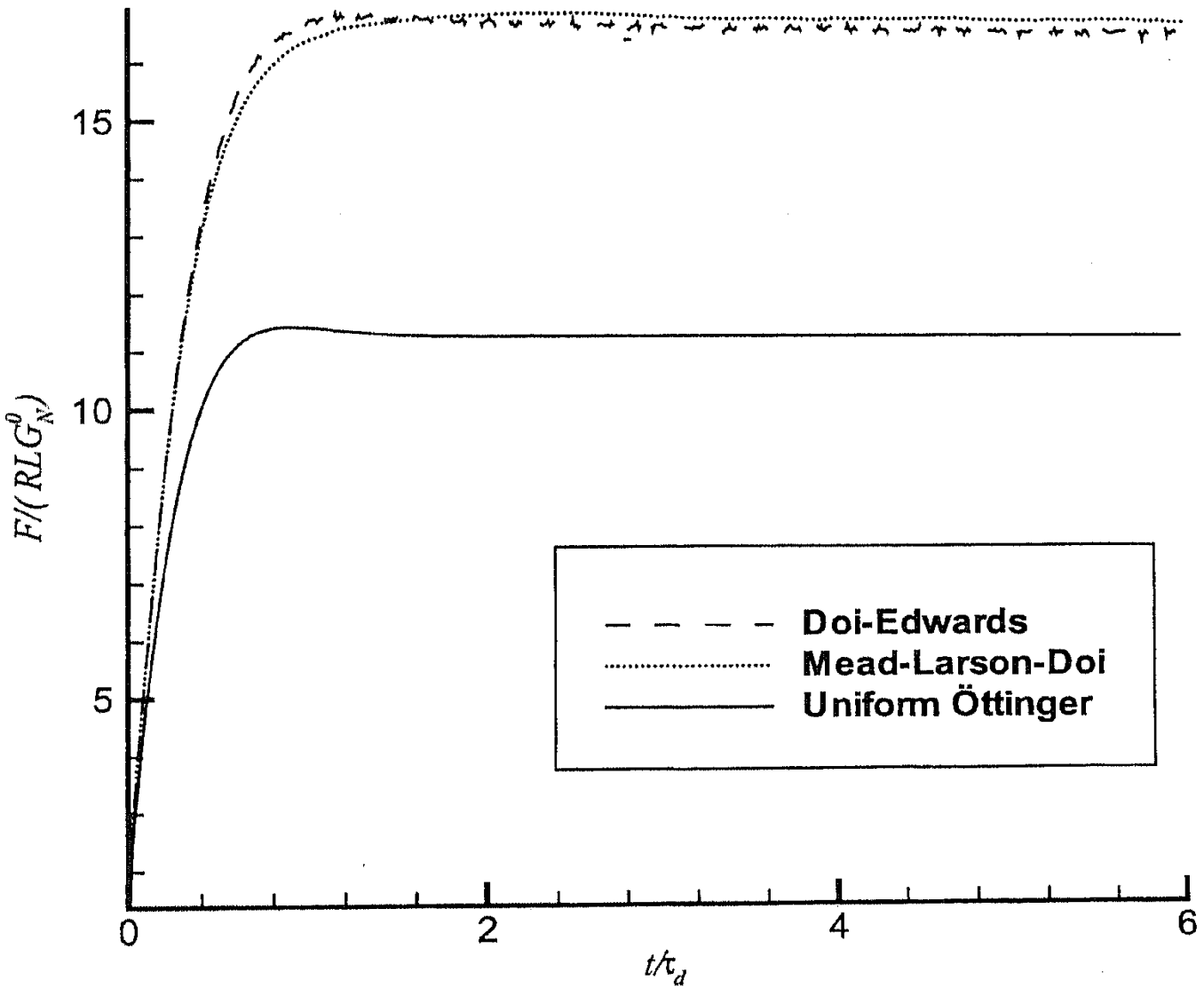

FIG. 14. Filow past a contined cylinder: Comparison of dimensionless drag force for DE and MLD models [Peters et al. $(2000 \mathrm{~b})]$ and UÖ model at $\mathrm{De}=0.3, L$ denotes the length of the cylinder. 


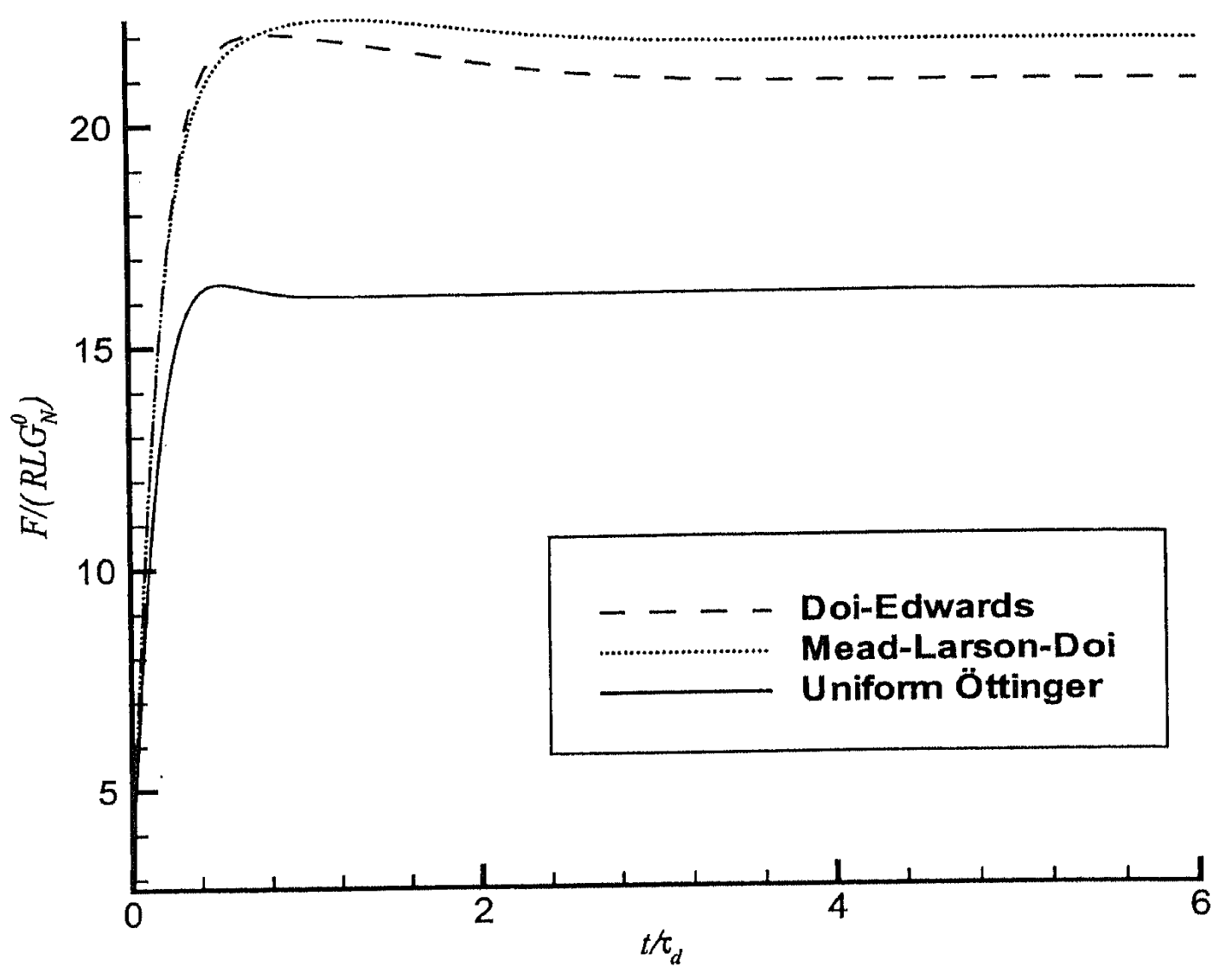

FIG. 15. Flow past a confined cylinder: Comparison of dimensionless drag force for DE and MLD models [Peters et al. $(2000 \mathrm{~b})]$ and UÖ model at $\mathrm{De}=0.6 . L$ denotes the length of the cylinder.

stress are plotted along the axis of symmetry and on the cylinder surface. Figure 13 shows that at Deborah numbers of 0.3 and 0.6 the departure from unity of the stretch parameter $\lambda$ for the UÖ and MLD models on the cylinder surface is very small (less than $5 \%$ difference between the maximum and equilibrium values for the MLD model) with the stretch in the UÖ model even smaller than that of the MLD model. The extra contribution to the elastic stress due to chain stretching is, therefore, expected to be comparatively small and the stress-orientation tensor relations (12) and (19) essentially collapse to that of the DE model (5). This does not mean that the drags on the cylinder computed with the three models will be even approximately the same, however. Certainly, and the same was observed by Peters et al. (2000b), low Deborah numbers, and a solvent viscosity $\eta_{s}=0.05 G_{N}^{0} \tau_{d}$ tend to mask the difference between the single-mode $\mathrm{DE}$ model and the MLD model. However, as may be observed from Figs. 14 and 15 a slightly higher steady-state drag is found at $\mathrm{De}=0.3$ and 0.6 with the MLD model than for the single-mode DE model, due to the small amount of tube stretching. We remark here that the drags computed by Peters et al. $(2000 \mathrm{~b})$, and presented in Figs. 4 and 6 of their paper, were for only half a cylinder [Peters (2002)]. Accordingly, we have doubled all their drag values in our discussion of the results.

Clearly, the explanation for the significant difference visible in Figs. 14 and 15 between the computed steady-state drag values for the UÖ model and the other two models must lie other than in the amount of tube stretch present. The key to the explanation lies in a comparison of the FP equations (11) and (17) for the single-mode DE and UÖ models. With $\lambda \approx 1, \dot{\lambda}_{\text {dissip }} \approx 0$, and a noteworthy difference in this case between the two FP equations is seen to be the presence of the diffusion term $\left(\delta_{1} / 6 \tau_{d}\right) \partial / \partial \mathbf{u} \cdot \partial \psi / \partial \mathbf{u}$ in Eq. (17). This double reptation term in the UÖ model introduces an additional relaxation 


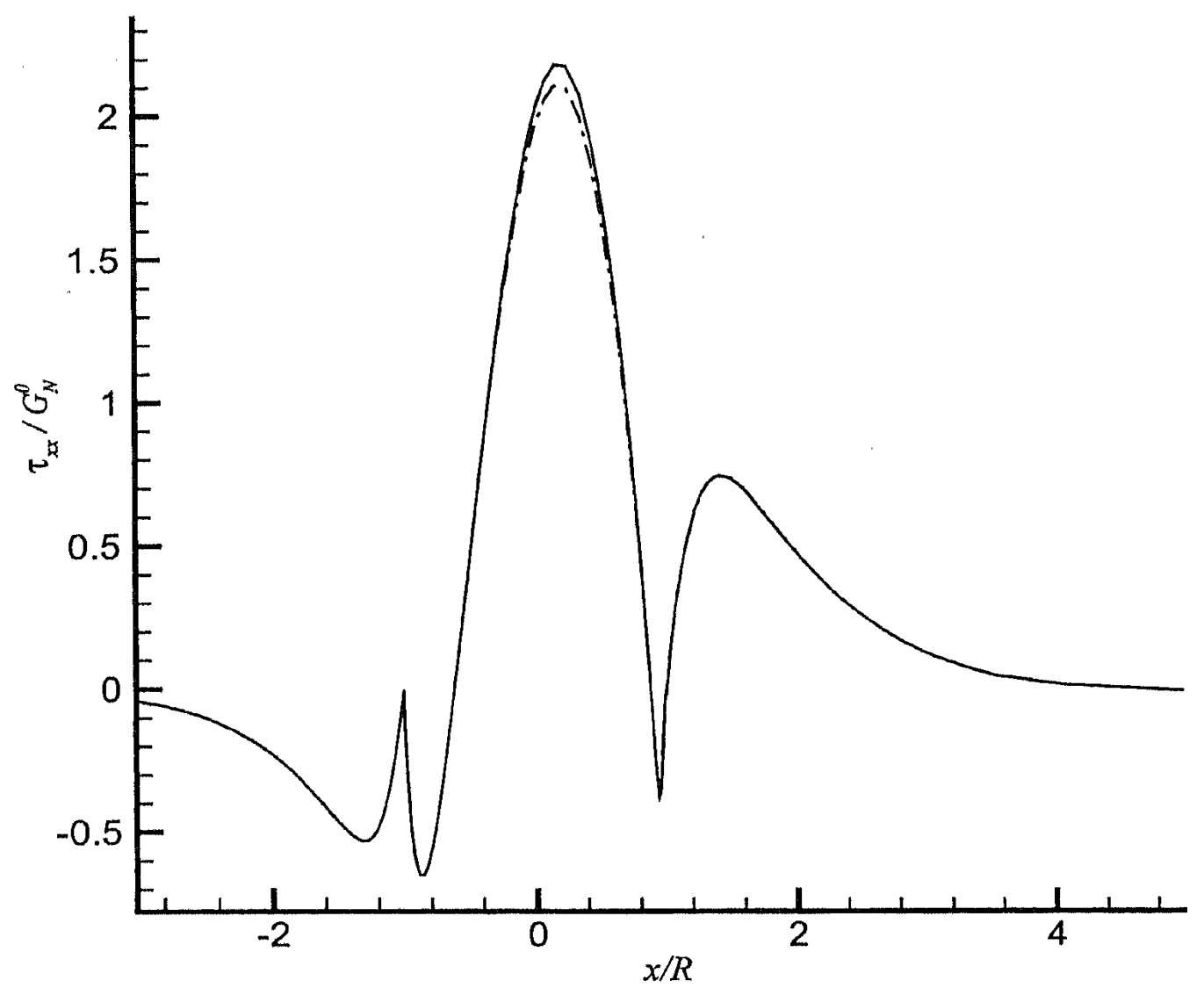

FIG. 16. Hlow past a confined cylinder: Nondimensionalized stress $\tau_{x x} / G_{N}^{0}$ along the axis of symmetry and on the cylinder surface $\left(-1 \leq x / R \leqslant 1\right.$ ) for UÖ model at $\mathrm{De}=0.6 . \eta_{s}=0, N_{u}=N_{s}=10$. Solid curve: $\beta / G_{N}^{0} \tau_{d l}=0.5, \Delta t / \tau_{d}=0.005$, and $N_{a x}=25$. Dashed and dotted curve: $\beta / G_{N}^{0} \tau_{d}=1, \Delta t / \tau_{d}=0.01$, and $N_{a x}=50$.

mechanism for the polymer chains and results in a primitive relaxation time a half that of the DE model. Chains in the UÖ model have the possibility of escaping their tubes more quickly than those in the single-mode DE model. Hence, they may escape high orientation with the flow field and as a consequence normal stresses are lower and so is the drag. As the flow rate is increased (going from Fig. 14 to 15) with $\tau_{d}$ unaltered, both sets of drag values increase in magnitude, but those for the DE and MLD models remain larger than that for the UÖ model.

\section{Computation for zero solvent viscosity: flow of a melt}

Simulations of the UÖ fluid in the case of a zero solvent viscosity necessitates modification of the numerical treatment of the linear momentum equation (22). As a generalization of the approach adopted in the previous subsection we now introduce a variant of the discrete elastic-viscous split-stress gradient (DEVSS-G) scheme of Liu et al. (1998) in the form

$$
\nabla p^{j}-\left(\eta_{s}+\beta\right) \nabla^{2} \mathbf{v}^{j}=\nabla \cdot\left[\boldsymbol{\tau}^{j}-\beta\left(\mathbf{G}^{j-1}+\mathbf{G}^{j-1 T}\right)\right]
$$

where $\beta$ is a viscosity term. $\mathbf{G}$ is a continuous second-order tensor, which is obtained by filtering the velocity gradient tensor $\nabla \mathbf{v}$ in each spectral element using an exponential filter [see Majda et al. (1978)] and then replacing the component values on elemental interfaces by the average values computed there from the adjoining elements. We note that the addition of the elliptic stabilization terms on both sides of Eq. (38) does not modify the continuous linear momentum equations, and furthermore, that in the limit $\beta \rightarrow 0$ we recapture our original scheme (22). 


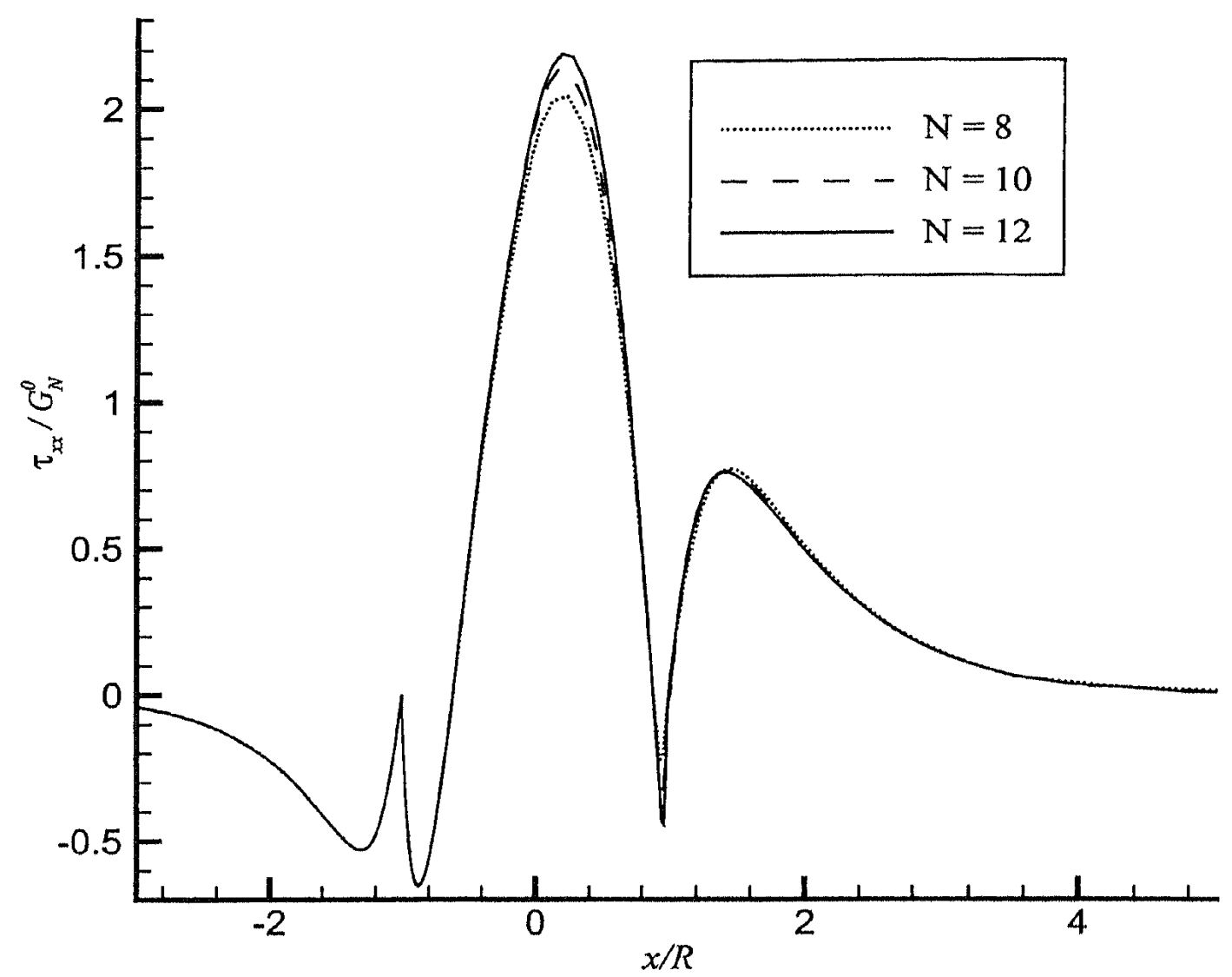

FIG. 17. Flow past a confined cylinder: Non-dimensionalized stress $\tau_{x x} / G_{N}^{0}$ along the axis of symmetry and on

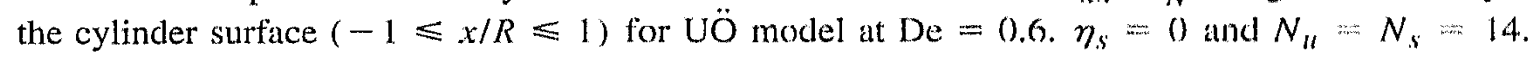

In validation of our numerical method for zero solvent viscosity, we present in Figs. 16 and 17 some results of convergence studies at $\mathrm{De}=0.6$. In Fig. 16 profiles of $\tau_{x x}$ (the most sensitive component of the elastic stress) along the axis of symmetry and on the cylinder surface are shown. We see that choosing two different values of $\beta$ has little effect on the computed elastic stress, with only a small discrepancy visible near the peak value on the cylinder surface. Convergence with spatial mesh refinement $(N=8,10$, and 12) is evident from Fig. 17 and convergence with refinement in configurational space may be deduced by comparing the $N=10$ plots from Figs. 16 and 17. The nondimensionalized drag, being an integrated quantity (and, therefore, averaged and smoothed, in some sense) is considerably less sensitive than the components of the elastics stress to changes in the resolution in configurational and real space, and this is apparent from Fig. 18 where for $\eta_{s}=0$ very close agreement at all times is seen between simulations on a coarse $\left(N_{u}=N_{s}=N=10\right)$ and a fine $\left(N_{u}=N_{s}=14, N=12\right)$ mesh.

Having established the reliability of our DEVSS-G scheme, we now proceed to consider the effects on the drag, chain stretch, and Cauchy stress of selecting $\eta_{s}$ to be zero (melt) or nonzero (concentrated polymer solution). Consistent with our observations from Figs. 13-15 that chain stretching effects on the elastic stress are comparatively small, we note from Fig. 18 that a nonzero solvent viscosity results in a higher drag on the cylinder than in the zero solvent viscosity case but that this must be due to the viscous stresses rather than to chain contour stretching. This assertion is substantiated in Fig. 19 where for both zero and nonzero solvent viscosities the maximum stretch on the cylinder is less than $6 \%$ of the equilibrium value. 


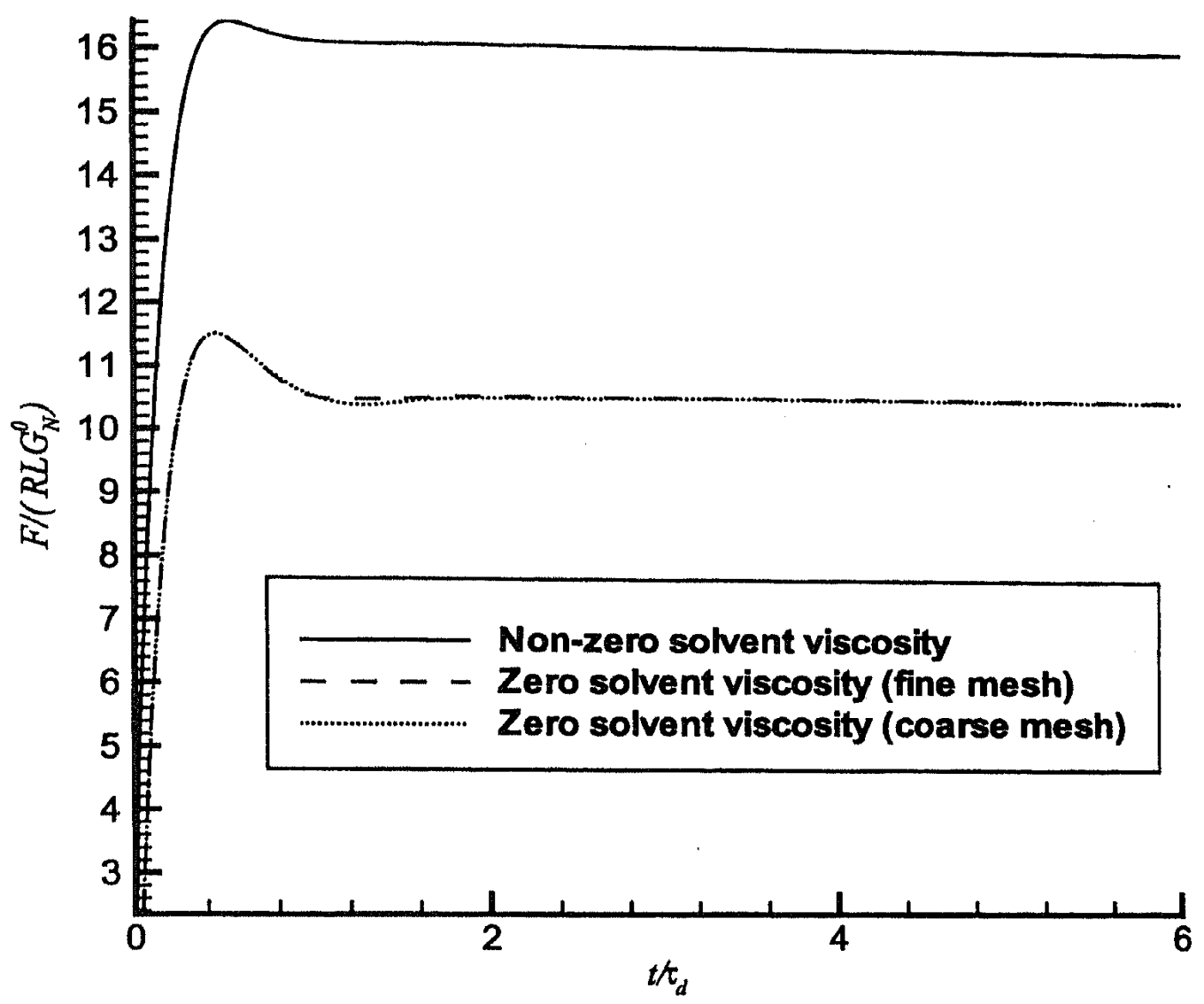

FIG. 18. Flow past a confined cylinder: Dimensionless drag force for UÖ model at $\mathrm{De}=0.6$. Solid curve: $\eta_{s} / G_{N}^{0} \tau_{d}=0.05, \beta=0$. Dashed and dotted curves: $\eta_{s}=0, \beta / G_{N}^{0} \tau_{d}=1$. For the nonzero solvent viscosity results and the $\eta_{s}=0$ fine mesh computation $N_{u}=N_{s}=14$ and $N=12$. For the $\eta_{s}=0$ coarse mesh computation $N_{u}=N_{s}=N=10 . L$ denotes the length of the cylinder.

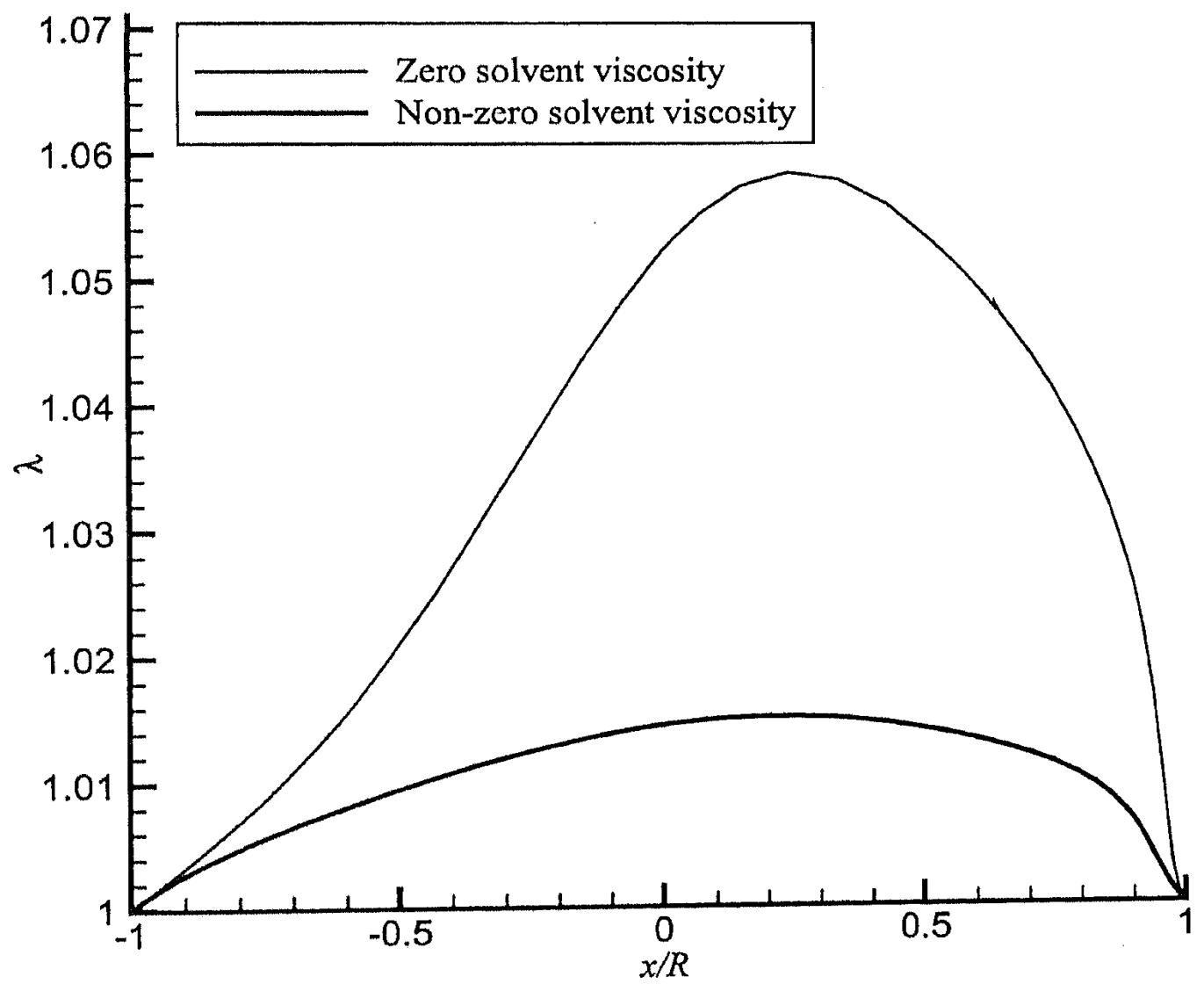

FIG. 19. Flow past a confined cylinder: Stretch parameter $\lambda$ on the cylinder surface for UÖ model at $\mathrm{De}=0.6$. $N_{u}=N_{s}=14$, and $N=10$. Thick curve: $\eta_{s} / G_{N}^{0} \tau_{d}=0.05, \beta=0$. Fine curve: $\eta_{s}=0, \beta / G_{N}^{0} \tau_{d}=1$. 


\section{CONCLUSIONS}

In this paper, we have described a new high-order FP-based method for the numerical simulation of the UÖ model [Öttinger (1999)] in complex flows. Double reptation and differences in the relaxation spectra play key roles in explaining differences observed in the drag behavior between the UÖ model and those of Doi and Edwards (1978a, 1978b, 1978c) and Mead et al. (1998) in the confined cylinder benchmark problem.

The method is found to be significantly cheaper (for the same level of accuracy) than the stochastic method of Fang et al. (2000) in startup homogeneous flows. The comparative cheapness of our method should make it a suitable candidate for simulating threedimensional flows and for use with models having higher-dimensional configurational spaces.

\section{ACKNOWLEDGMENTS}

The authors wish to thank Frank Peters and Martien Hulsen for making available data from their paper [Peters et al. (2000b)]. Discussions held with Frank Peters proved to be both interesting and illuminating. One of the authors (A.L.) was supported by the Swiss National Science Foundation, Grant No. 2100-057119.

\section{References}

Armstrong, R. C., R. Nayak, I. Ghosh, and R. A. Brown, "The use of kinetic theory and microstructural models in the analysis of complex flows of viscoelastic liquids," in Proceedings of the /2th Internutional Congress on Rheology, 18-23 August, 1996, edited by Aït-Kadi, A., J. M. Dealy, D. F. James, and M. C. Williams (Laval University, Québec, 1996).

Canuto, C., M. Y. Hussaini, A. Quarteroni, and T. A. Zang, Spectral Methods in Fluid Dynamics (Springer, Berlin, 1988).

Chauvière, C., and R. G. Owens, "A new spectral element method for the reliable computation of viscoelastic flow," Comput. Methods Appl. Mech. Eng. 190, 3999-4018 (2001).

de Gennes, P. G., "Reptation of a polymer chain in the presence of lixed obstacles," J. Chem. Phys. 55, $572-579$ (1971).

des Cloizeaux, J., "Double reptation versus simple reptation in polymer melts," Europhys. Lett. 5, 437 -..442 (1988).

Doi, M., and S. F. Edwards, "Dynamics of concentrated polymer systems: Brownian motion in the equilibrium state," J. Chem. Soc., Faraday Trans. 74, 1789-1801 (1978a).

Doi, M., and S. F. Edwards, "Dynamics of concentrated polymer systems: Molecular motion under llow," J. Chem. Soc., Faraday Trans. 74, 1802-1817 (1978b).

Doi, M., and S. F. Edwards, "Dynamics of concentrated polymer systems: The constitutive equation," J. Chem. Soc., Faraday Trans. 74, 1818-1832 (1978c).

Fan, X.-J., "Viscosity, first normal-stress coefficient, and molecular stretching in dilute polymer solutions," $J$. Non-Newtonian Fluid Mech. 17, 125-144 (1985a).

Fan, X.-J., "Viscosity, first normal-stress coefficients, and molecular stretching in concentrated solutions and melts," J. Non-Newtonian Fluid Mech. 17, 251-265 (1985b).

Fan, X.-J., "A numerical investigation of the hydrodynamic interaction for Hookean dumbbell suspensions in steady state shear flow," J. Chem. Phys. 85, 6237-6238 (1985c).

Fan, X., "Molecular models and flow calculation: I. The numerical solutions to multibead-rod models in inhomogeneous flows," Acta Mech. Sin. 5, 49-59 (1989a).

Fan, X. "Molecular models and flow calculations: II. Simulation of steady planar flow," Acta Mech. Sin. 5, $216-226(1989 b)$.

Fang, J., M. Kröger, and H. C. Öttinger, "A thermodynamically admissible reptation model for fast tlows of entangled polymers. II. Model predictions for shear and extensional flows," J. Rheol. 44, 1243-1317 (2000).

Gigras, P. G., and B. Khomami, "Adaptive configuration helds: A new multiscale simulation technique for reptation-based models with a stochastic strain measure and local variations of life span distribution," J. Non-Newtonian Fluid Mech. 108, 99--122 (2002). 
Halin, P., G. Lielens, R. Keunings, and V. Legat, "The Lagrangian particle method for macroscopic and micro-macro viscoelastic flow computations," J. Non-Newtonian Fluid Mech. 79, 387-403 (1998).

Hassager, O., private communication (2002).

Hua, C. C., and J. D. Schieber, "Segment connectivity, chain-length breathing, segmental stretch, and constraint release in reptation models. I. Theory and single-step strain predictions," J. Chem. Phys. 109, 10018-10027 (1998).

Hua, C. C., J. D. Schicber, and D. C. Venerus, "Segment connectivity, chain-length breathing, segmental stretch, and constraint release in reptation models. III. Shear flows," J. Rheol. 43, 701-717 (1999).

Hulsen, M. A., E. A. J. F. Peters, and B. H. A. A. van den Brule, "The deformation fields method for solving complex flows using integral constitutive equations," J. Non-Newtonian Fluid Mech. 98, 201-221 (2001).

Hulsen, M. A., A. P. G. van Heel, and B. H. A. A. van den Brule, "Simulation of viscoelastic flows using Brownian configuration fields," J. Non-Newtonian Fluid Mech. 70, 79-101 (1997).

Ianniruberto, G., and G. Marrucci, "On compatibility of the Cox-Merz rule with the model of Doi and Edwards," J. Non-Newtonian Fluid Mech. 65, 241-246 (1996).

Kahvand, H., "Strain Coupling Effects in Polymer Rheology," Ph.D. thesis, Illinois Institute of Technology (1995).

Laso, M., and H. C. Öttinger, "Calculation of viscoelastic flow using molecular models: the CONNFFESSIT approach," J. Non-Newtonian Fluid Mech. 47, 1-20 (1993).

Liu, A. W., D. E. Bornside, R. C. Armstrong, and R. C. Brown, "Viscoelastic flow of polymer solutions around a periodic, linear array of cylinders: Comparisons of predictions for microstructure and flow fields," J. Non-Newtonian Fluid Mech. 77, 153-190 (1998).

Maday, Y., A. T. Patera, and E. M. Ronquist, "The $P_{N}-P_{N-2}$ method for the approximation of the Stokes problem," Technical Report No. 92025, Laboratoire d'Analyse Numérique, Université Pierre et Marie Curie (1992).

Majda, A., J. McDonough, and S. Osher, "The Fourier method for nonsmooth initial data," Math. Comput. 32, $1041-1081$ (1978).

Marrucei, G., "Dynamics of entanglements: A nonlinear model consistent with the Cox-Merz rule," J. NonNewtonian Fluid Mech. 62, 279-289 (1996).

Marruci, G., and N. Grizizuti, "Fast flows of concentrated polymers-Predictions of the tube model on chain stretching," Gitz. Chim. Ital. 118, 179-185 (1988).

Mead, D. W., R. G. Larson, and M. Doi, "A molecular theory for fast flows of entangled polymers," Macromolecules 31, 7895-7914 (1998).

Nayak, R., "Molecular simulation of liquid crystal polymer flow: A wavelet-finite element analysis," Ph.D. thesis, Ml'T, Cambridge, MA (1998).

Öttinger, H. C., Stechastic: Processes in Polymeric Fluids (Springer, Berlin, 1996).

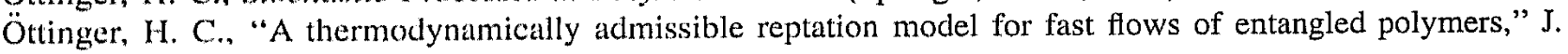
Rheol. 43, 1401-1493 (1999).

Öttinger, H. C., B. H. A. A. van den Brule, and M. A. Hulsen, "Brownian configuration fields and variance reduced CONNFFESSIT," J. Non-Newtonian Fluid Mech. 70, 255-261 (1997).

Owens, R. G., and T. N. Phillips, "Steady viscoelastic flow past a sphere using spectral elements," Int. J. Numer. Methods Eng. 39, 1517-1534 (1996).

Owens, R. G., and T. N. Phillips, Computational Rheology (Imperial College Press/World Scientific, Singapore, $2002)$.

Pearson, D. S., A. D. Kiss, L. J. Fetters, and M. Doi, "Flow-induced birefringence of concentrated polyisoprene solutions," J. Rheol. 33, 517-535 (1989).

Peters, E. A. J. F., M. A. Hulsen, and B. H. A. A. van den Brule, "Instationary Eulerian viscoelastic flow simulations using time separable Rivlin-Sawyers constitutive equations," J. Non-Newtonian Fluid Mech. $89,209-228(2000$ a).

Peters, E. A. J. F., A. P. G. van Heel, M. A. Hulsen, and B. H. A. A. van den Brule, "Generalization of the deformation field method to simulate advanced reptation models in complex flow," J, Rheol. 44, 811-829 $(2000 b)$.

Peters, E. A. J. F., private communication (2002).

Suen, J. K. C., Y. L. Joo, and R. C. Armstrong, "Molecular orientation effects in viscoelasticity," Annu. Rev. Filuid Mech. 34, $41-444$ (2002).

Tsenoglou, C.., "Viscoelasticity of binary homopolymer blends," Polym. Prepr. (Am. Chem. Soc. Div. Polym. Chem.) 28, 185-186 (1987).

van Heel, A. P. G., M. A. Hulsen, and B. H. A. A. van den Brule, "Simulation of the Doi-Edwards model in complex flow," J. Rheol. 43, 1239-1260 (1999).

Wapperom, P., R. Keunings, and V. Legat, "The backward-tracking Lagrangian particle method for transient viscoelastic Alows," J. Non-Newtonian Fluid Mech. 91, 273-295 (2000).

Warner, H. R., "Kinetic theory and rheology of dilute suspensions of finitely finitely extendible dumbbells," Ind. Eng. Chem. Fundam. 11, 379--387 (1972). 